\title{
Contribution à la connaissance des Nématodes de Sauriens Malgaches
}

\author{
Par Guilliermina CABALLERO R. \\ (Laboratoire de Zoologie (Vers) associé au C.N.R.S., Muséum National d'Histoire Naturelle, \\ 57, rue Cuvier. F. 75 - Paris-5")
}

\begin{abstract}
Résumé
Description de 15 nouvelles espèces ou sous-espèces de Nématodes parasites de Sauriens malgaches. Non compris les Serpents, il y a maintenant 54 espèces connues chez les Reptiles de l'Ile et les caractères particuliers de cette faune sont analysés.

\section{Summary}

Description of 15 new species or subspecies of parasitic Nematodes from Madagascar Lizards. Excluding the Snakes, there are now 54 species known in the Reptiles of the Island and the peculiar characteristics of this fauna are analysed.
\end{abstract}

Nous remercions vivement M. le Professeur B. Possompes d'avoir accepté de présider le jury de notre thèse.

Nous remercions également M. le Professeur G. Echalier d'avoir bien voulu faire partie de notre jury.

Nous exprimons notre très profonde et sincère reconnaissance à M. le Professeur A.-G. Chabaud qui a dirigé ce travail, en a guidé attentivement le développement, nous a conseillée avec un inlassable bienveillance, et nous a fait pofiter de sa très grande expérience.

Nous adressons nos vifs remerciements à M.-C. Dupuis, qui par ses précieux conseils nous a facilité la rédaction de ce travail.

M. E.-R. Brygoo et M.-Ch. Blanc ont bien voulu nous confier la plus grande partie de notre matériel d'étude. Qu'ils trouvent ici l'expressiin de notre très vive gratitude.

Nous tenons à exprimer également nos remerciements à $\mathrm{M}$. le $\mathrm{Pr}$ J. Guibé qui a eu l'extrême obligeance de nous préciser les déterminations des Reptiles malgaches.

Nous remercions $\mathbf{M}^{\mathrm{me}} \mathrm{N}$. Korbendau, $\mathbf{M}^{\mathrm{me}} \mathbf{R}$. Tcheprakoff et $\mathbf{M}^{\mathrm{me}} \mathbf{M}$. Weille pour leur collaboration technique, ainsi que tout le personnel du Laboratoire qui nous a prêté son concours. 


\section{INTRODUCTION}

Ce travail a été réalisé sur une collection de Nématodes parasites de Sauriens malgaches, provenant de nombreuses récoltes effectuées par M. E. R. Brygoo, Directeur de l'Institut Pasteur de Madagascar, par M. Ch. P. Blanc, de la Faculté des Sciences et Techniques de l'Université de Madagascar et par les Missions du Laboratoire de Zoologie (Vers) du Muséum National d'Histoire Naturelle de Paris.

L'étude, effectuée sur les Nématodes provenant d'environ 150 Sauriens d'espèces variées, comprend : la description de 15 nouvelles espèces et d'une nouvelle sous-espèce, et des données sur la fréquence et la spécificité des espèces.

L'étude de Nématodes de Reptiles malgaches ayant été réalisée pour les Chéloniens par Petter, 1966, pour les Crocodiliens par Chabaud et Caballero, 1966 et pour les Chaméléonidés par Brygoo, 1963, nous disposerons au terme de notre étude, et pour la première fois, d'un aperçu complet sur les Nématodes de Reptiles malgaches autres que les Serpents, lesquels ont une faune de Nématodes encore mal connue et vraisemblablement très différente de celle des autres Reptiles.

Nous chercherons, en nous appuyant sur l'ensemble de ces documents, à tirer des conclusions générales et à préciser la singularité de cette faune selon les groupes d'hôtes, d'une part, selon les groupes de parasites, d'autre part.

Le matériel étudié est déposé au Muséum National d'Histoire Naturelle de Paris, Laboratoire de Zoologie (Vers).

\section{Nématodes de Geckonoidea}

Nous avons examiné les Nématodes provenant de Geckonidae appartenant aux genres Geckolepis, Hemidactylus, Phelsuma et Phyllodactylus, et les Nématodes d'Uroplatidae appartenant au genre Uroplatus.

\section{A. - Etude des espèces}

1) SKRJABINELAZIA ORNATA Chabaud, Caballero et Brygoo 1965 (Seuratidae).

Cette espèce (fig. 1: A, B, C, D, E, F, G, H, I), décrite récemment de l'estomac et du rectum du Geckonidae Phelsuma lineatum Gray de la région de la Mandraka a été retrouvée une troisième fois chez le même hôte $(1009 \mathrm{~F}, 1010 \mathrm{~F}$ et $1012 \mathrm{~F})$ dans la même région, puis une quatrième fois chez un lézard Zonosaurus madagascariensis (Gray) (469 G) de Mahabo (Côte Est). 

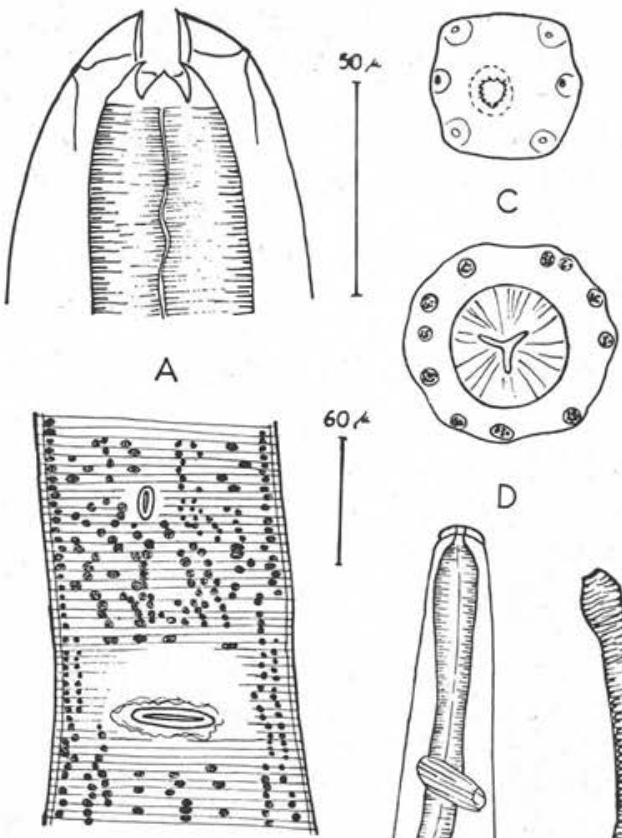

B
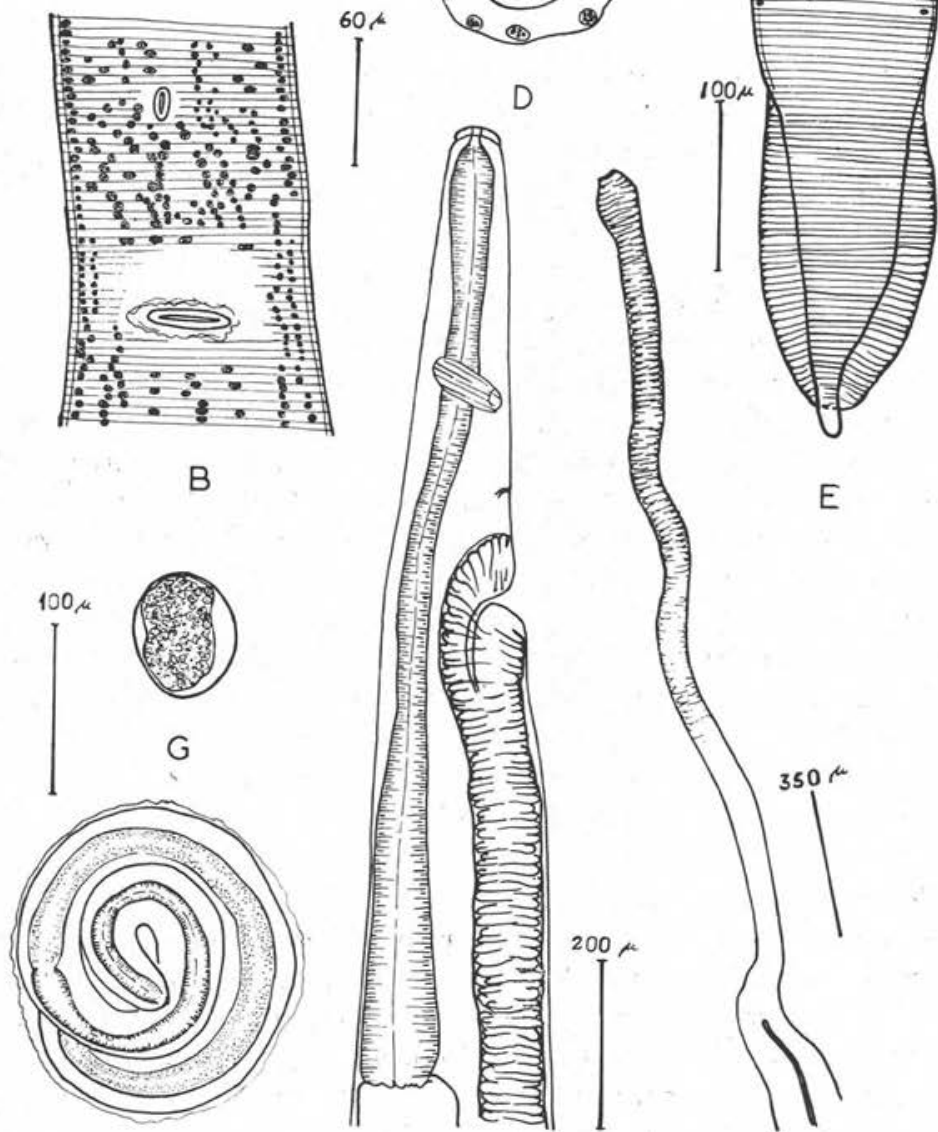

$\mathrm{H}$

F

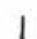

Fı́.. 1. - Skrjabinelazia ornata Chabaud, Caballero R. et Brygoo, 1964. Femelle. A, extrémité antérieure, vue dorsale. B, pore excréteur et vulve, vue ventrale. C, tête, vue apicale. D, coupe optique de la région antérieure de l'œsophage. E, queue, vue ventrale. F, région œsophagienne, vue latérale. $\mathrm{G}$, œuf immature. H, larve mûre, I, ovéjecteur 
Les mâles ne sont pas connus, mais les femelles paraissent avoir des affinités avec les deux espèces décrites dans le genre Skrjabinelazia Sypliaxov, 1930, et la forme malgache a été classée provisoirement dans ce genre. Elle pourrait ainsi être rapprochée d'espèces parasites de Lacertidae, d'une part de l'espèce russe $S$. taurica Sypliaxov, 1930, parasite de Lacerta taurica Pallas, et d'autre part de l'espèce chinoise $S$. hoffmani Li, 1934, parasite de Eremias argus Peters.

Comme tous les Seuratoidea, les espèces du genre Skrjabinelazia présentent des caractères synthétiques et semblent caractéristiques de faunes archaïques.

2) PHARYNGODON BREVIBURSATA n. sp., (Oxyuridae).

\section{Matériel étudié.}

Les spécimens types, qui portent l'étiquette $538 \mathrm{G}$, ont été récoltés dans le rectum d'un Geckolepis sp., de Mahabo. Des spécimens de la même espèce ont été retrouvés chez un autre Geckolepis sp. (521 G), chez Geckolepis maculata Peters (668 G) de Nossi-Bé et chez les Phelsuma sp. (508 G, 518 G) de Mahabo.

\section{Deseription.}

De forme allongée, les parasites possèdent un appendice caudal effilé. La cuticule blanchâtre, à stries transversales fines, s'élargit latéralement et forme des ailes courtes qui prennent naissance un peu en avant de l'anneau nerveux.

Mâle: corps long de $1,94 \mathrm{~mm}$ sur $150 \mu$ de large. Extrémité antérieure étroite et plate. La tête porte quatre papilles submédianes et deux amphides latérales. La bouche, circulaire, est délimitée par un rebord festonné (fig. 2, G). Esophage court, longueur totale : $195 \mu$ (corpus : $120 \mu$, isthme : $25 \mu$ et bulbe : $50 \mu$ ). Anneau nerveux et pore excréteur situés respectivement à $110 \mu$ et $535 \mu$ de l'apex (fig. 2, F).

L'extrémité postérieure est ornée d'une bourse caudale bien développée, circulaire, de $80 \mu$ de longueur sur $90 \mu$ de largeur. Trois paires de papilles, dont la première préanale, sessile, se trouve déplacée vers les côtés latéraux du corps ; la seconde paire adanale, est pédonculée et bifide ; la troisième paire post-anale est également pédonculée (fig. 2, H). Le cône génital, saillant, est recouvert ventralement par une membrane très fine et transparente (fig. 2, J). Spicule et gubernaculum absents. L'appendice caudal, très effilé, est long de $80 \mu$.

Femelle: corps long de $3,1 \mathrm{~mm}$ sur $300 \mu$ de large. Les áiles latérales naissent en avant de l'anneau nerveux et se terminent à mi-chemin entre l'anus et la pointe caudale. Chacune d'entre elles est constituée de deux feuillets minces et très étroits, soudés tout au long de la ligne latérale (fig. 2, C). Extrémité céphalique petite. Six lobes entourent la bouche, les deux latéraux paraissent être subdivisés et portent les amphides, les lobes dorsaux et ventraux portent chacun une petite papille (fig. 2, B). L'œsophage total mesure $285 \mu$ (corpus : $190 \mu$, isthme : $20 \mu$, bulbe : $75 \mu$ ).

Anneau nerveux, pore excréteur et vulve situés respectivement à $120 \mu, 460 \mu$ et $500 \mu$ de l'apex.

L'ovéjecteur mesure 1,05 mm (fig. 2, D) ; il débute par une portion musculaire longue de $550 \mu$, suivie d'une partie à parois minces de $200 \mu$, et enfin d'une section à 


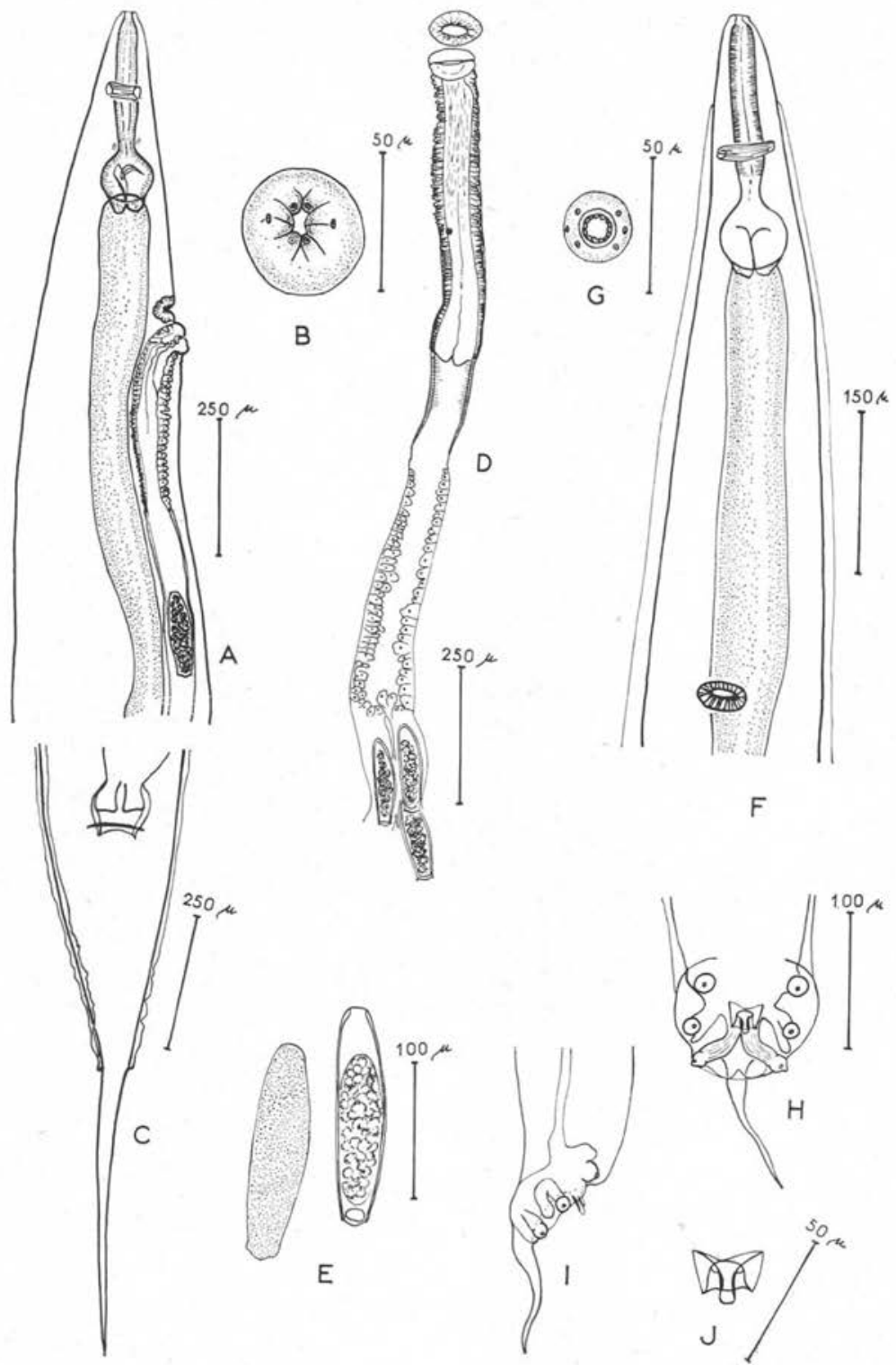

Fig. 2. - Pharyngodon brevibursata n sp. A, extrémité antérieure de la femelle, vue latérale. B, tête de la femelle, vue apicale. C, extrémité postérieure de la femelle, vue ventrale. D. ovéjecteur. E, cufs. F, extrémité antérieure du mâle, vue ventrale. G, tête du mâle, vue apicale. H, extrémité postérieure du mâle, vue ventrale. I, extrémité postérieure du mâle, vue latérale. J, cône génital 
larges cellules. Les œufs sont operculés à un de leurs pôles, leur coque est granuleuse, ils mesurent $137 \mu$ de long sur $32 \mu$ de large (fig. 2, E). La queue est longue de $825 \mu$ et se termine par un appendice fin (fig. 2, C).

\section{Discussion.}

L'espèce appartient au genre Pharyngodon Diesing, 1861. Elle se place dans le groupe d'espèces à mâles pourvus de grandes ailes caudales, soutenues par les papilles incluses dans leur épaisseur, de papilles ad-anales denses à l'apex et d'une pointe caudale relativement courte ; à femelles à pointe caudale non épineuse, c'est-à-dire : Pharyngodon hindlei Thapar, 1925, P. cesarpintoi Pereira, 1935, P. mamillatus (Linstow, 1897), $P$. australe Johnston et Mawson, 1940. Les espèces décrites plus récemment, comme P. morgani Fitzsimmons, 1961, parasite du Scincidae Mabuya striata Peters, du Nyassaland, s'éloignent des précédentes parce que la troisième paire de papilles caudales se trouve hors des ailes caudales.

- L'espèce $P$. hindlei Thapar, 1925, parasite du Scincidae Tiliqua scincoides (White) en Australie, diffère de nos spécimens par la présence d'un spicule et par la forme de la queue chez la femelle.

- P. cesarpintoi Pereira, 1935, parasite du Taejidae Cnemidophorus lemniscatus (Daudin) au Brésil, est différent parce que la deuxième paire de papilles caudales est longuement pédonculée et que la queue de la femelle est conique, courte et fine.

- P. mamillatus (Linstow, 1897), parasite des Scincidae Eumeces schneideri (Daudin) (= Plestiodon aldrovandi) d'Egypte, Eumeces algeriensis Peters et Eumeces sp., de Madagascar, du Maroc et d'Espagne, diffère de nos exemplaires par les caractères suivants : les ailes prennent naissance juste en arrière du bulbe cesophagien et s'élargissent un peu en avant du cloaque ; la bourse caudale en forme de losange montre la paire de papilles ad-anales longuement pédonculée et ramifiée en deux à quatre digitations irrégulières.

- P. australe Johnston et Mawson, 1942, parasite du Scincidae Tiliqua scincoides (White) en Australie, diffère de notre matériel par la vulve et le pore excréteur, qui se trouvent au niveau du bulbe œsophagien, et par les œufs, plus grands.

Nos spécimens, ne pouvant être assimi'és à aucune des espèces du même groupe, paraissent donc constituer une espèce nouvelle, que nous nommons Pharyngodon brevibursata $\mathrm{n}$. sp.

3) PARATHELANDROS DOSSAE n. sp., (Oxyuridae).

\section{Matériel étudié.}

Le matériel que nous étudions se compose d'un mâle et d'une dizaine de femelles qui portent les étiquettes $975 \mathrm{~F}$ et $984 \mathrm{~F}$, parasites du Geckonidae Phelsuma lineatum Gray de Périnet.

\section{Description.}

Ces parasites sont fusiformes, à extrémité antérieure arrondie, et se terminent par 
un appendice caudal long et fin. Ils sont recouverts d'une cuticule fine et transparente. Les femelles ne possèdent pas d'ailes latérales.

Mâle: il mesure 2,2 $\mathrm{mm}$ de longueur sur $350 \mu$ de largeur. Les ailes latérales naissent en avant de l'anneau nerveux et se prolongent jusqu'au niveau de la première paire de papilles génitales où elles prennent leur plus grande largeur. L'extrémité antérieure est légèrement arrondie et étroite. L'œsophage court mesure au total $275 \mu$; il se termine par un bulbe subsphérique, peu musculeux. L'intestin est large de $90 \mu$ au niveau du pore excréteur. L'anneau nerveux et le pore excréteur sont situés respectivement à $100 \mu$ et $520 \mu$ de l'apex (fig. 3, A). Extrémité postérieure légèrement dilatée au niveau du cloaque. Absence d'ailes caudales. Trois paires de papilles, une pré-cloacale et deux post-cloacales (la seconde paire post-cloacale est placée ventralement sur l'appendice caudal). Le cône génital est renforcé par un épaississement cuticulaire chitineux. Devant le cloaque on observe une languette membraneuse (fig. 3, D, E). Spicule et gubernaculum absents. Queue très longue : $425 \mu$.

Femelle: elle mesure $9,4 \mathrm{~mm}$ de longueur sur $220 \mu$ de largeur au niveau de la vulve. Tête à grande bouche arrondie. Quatre papilles submédianes et deux amphides. En profondeur, trois dents œsophagiennes (fig. 3, B, C). Esophage court de $360 \mu$ de longueur (bulbe compris). Bulbe arrondi, faiblement musculeux. Anneau nerveux et pore excréteur respectivement situés à $110 \mu$ et $570 \mu$ de l'apex (fig. 3, F). Nous avons observé une partie du système excréteur, en particulier deux canaux latéraux antérieurs et deux canaux latéraux postérieurs qui débouchent dans une vésicule excrétrice à parois minces s'ouvrant à l'extérieur par le pore excréteur, large, et situé juste en avant de la vulve. Cette dernière est largement ouverte $(75 \mu$ de largeur) et est située à $620 \mu$ de l'extrémité antérieure. L'ovéjecteur est formé d'un long tube à parois peu musculeuses ; les deux ovaires sont situés au milieu du corps. L'utérus est rempli d'œufs, ovoïdes, jaunâtres, à protoplasme granuleux, de $190 \mu$ de long sur $45 \mu$ de large (fig. 3, H). Queue longue de 1,19 mm, pourvue d'un appendice, long, fin et épineux (fig. 3, G).

\section{Discussion.}

L'espèce se distingue de toutes les espèces connues du genre par la queue de la femelle, dont la partie terminale est un filament plus court que la partie conique et qui porte quelques petites épines.

Il est intéressant de noter que les formes apparemment les plus proches sont confinées en Afrique orientale et méridionale.

De plus :

- Chez Parathelandros megalocerca (Skrjabin, 1916) Skrjabin, Schikhobalova et Lagodovskaja, 1960, parasite d'un Gecko sp., d'Afrique orientale, les ailes latérales du mâle sont très dilatées dès la région œsophagienne.

- Chez P. mabuyae (Sandground, 1936) Skrjabin, Schikhobalova et Lagodovskaja, 1960, parasite du Scincidae Mabuya varia varia Peters en Ouganda, le mâle porte une frange précloacale et les œufs sont brusquement tronqués à l'extrémité.

- Chez P. mabuiensis (Malan, 1939) Skrjabin, Schikhobalova et Lagodovskaja, 


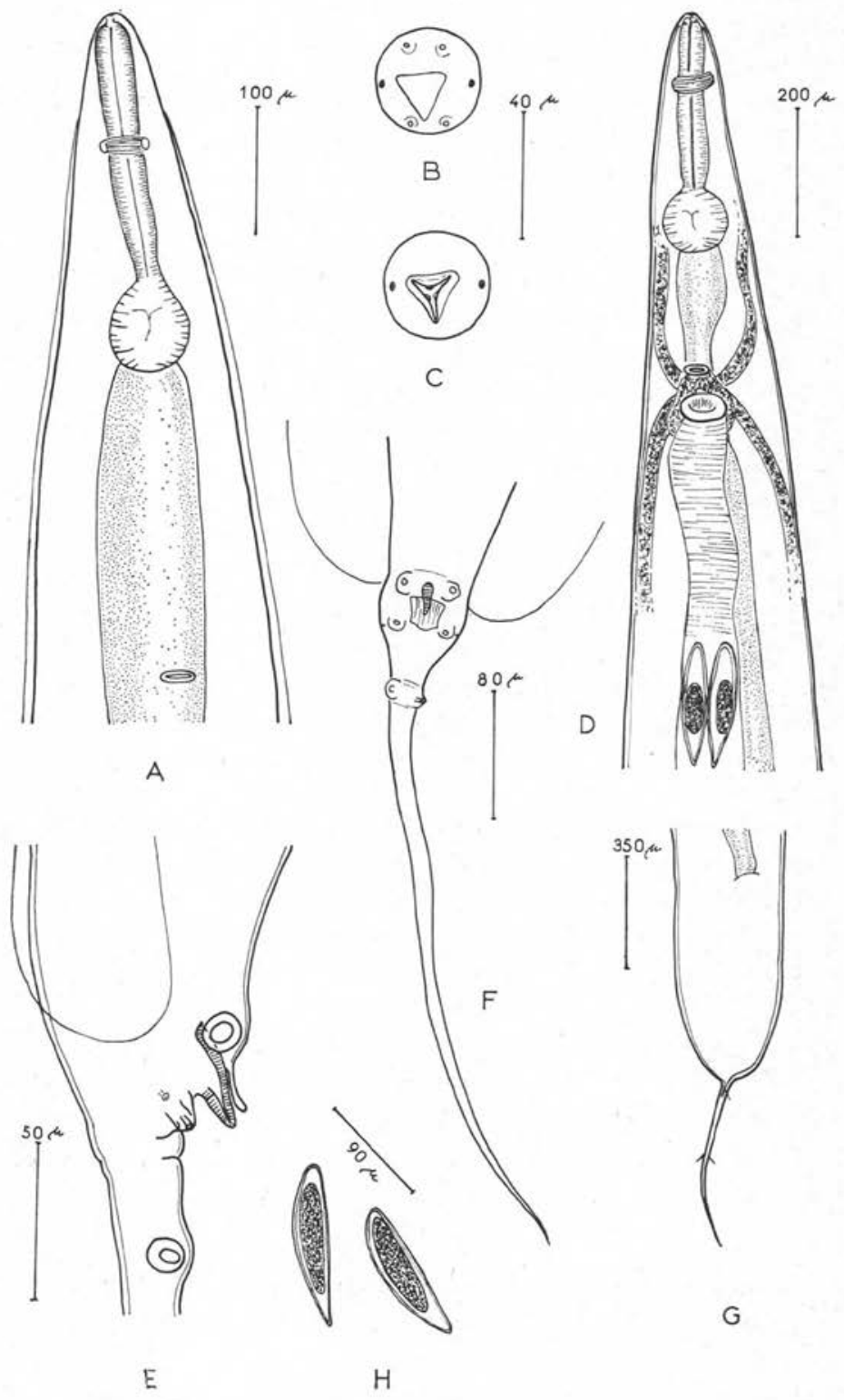

Fig. 3. - Parathelandros dossae n. sp. A, extrémité antérieure du mâle, vue ventrale. B, tête de la femelle, vue apicale superficielle. C, tête de la femelle, vue apicale profonde. D, extrémité antérieure de la femelle, vue ventrale. E, extrémité postérieure du mâle, vue latérale. $\mathrm{F}$, extrémité postérieure du mâle, vue ventrale. G, extrémité postérieure de la femelle, vue ventrale. H, œufs 
1960, parasite du Scincidae Mabuya trivittata (Cuvier) en Afrique du Sud, la pointe caudale est relativement courte.

L'espèce malgache peut donc être considérée comme nouvelle et nous la nommons Parathelandros dossae n. sp., en respectueux témoignage d'estime à Miss Mildred A. Doss.

4) PARATHELANDROS OVOCAUDATUS n. sp. (Oxyuridae).

\section{Matériel étudié.}

Nous possédons trois mâles et plusieurs femelles, qui portent les étiquettes $1017 \mathrm{~F}$, $1018 \mathrm{~F}$ et $1019 \mathrm{~F}$, parasites du rectum du Geckonidae Phelsuma mutabilis (Grandidier) de Antimora. Nous les avons aussi retrouvés chez Phelsuma sp. (572 E) d'Ambavaniasy.

\section{Description.}

Le corps des spécimens est fusiforme, leur extrémité antérieure est légèrement arrondie et la postérieure se termine par une longue pointe aiguë. Les ailes latérales prennent naissance en avant de l'anneau nerveux chez la femelle; chez le mâle, elles sont très étroites et se terminent au niveau de la première paire de papilles caudales. L'extrémité céphalique, en vue apicale, n'a été étudiée que chez la femelle.

Mâle: corps petit et grêle, mesurant 1,6 mm de longueur sur $250 \mu$ de largeur, recouvert d'une cuticule fragile et translucide. L'extrémité antérieure très étroite présente une courte cavité buccale, suivie de l'œsophage, long de $160 \mu$ et large de $35 \mu$, qui se termine par un bulbe subsphérique de $80 \mu$ sur $75 \mu$. L'intestin est large de $70 \mu$ au niveau du pore excréteur (fig. 4, E). Anneau nerveux et pore excréteur situés respectivement à $90 \mu$ et $350 \mu$ de l'apex.

Extrémité postérieure sans ailes caudales, terminée par une queue conique et pointue de $400 \mu$ de longueur. Autour du cloaque saillant se trouvent deux paires de papilles, une pré-cloacale et une autre post-cloacale. Une troisième paire est située au début de l'appendice caudal (fig. 4, F, G). Spicule et gubernaculum absents.

Femelle: corps long de $2 \mathrm{~mm}$ et large de $150 \mu$ au niveau de la vulve. Extrémité antérieure étroite. La tête porte quatre papilles submédianes peu saillantes et deux amphides latérales ; autour de la bouche, on observe six petites lèvres arrondies. En profondeur, au fond de la petite cavité buccale, on distingue un anneau chitinoïde. Une membrane ferme la cavité œsophagienne. La lumière de l'œsophage est bordée par un épaississement chitinoïde qui, au niveau de la cavité œsophagienne, forme trois dents fines, faisant saillie (fig. 4, B, C). L'œsophage long de $210 \mu$ et large de $40 \mu$ se termine par un bulbe sphérique de $80 \mu$ de diamètre. L'anneau nerveux, le pore excréteur et la vulve se trouvent respectivement à $125 \mu$ et $370 \mu$ d l'apex. L'appareil excréteur semble avoir une configuration identique à celle de l'espèce $P$. dossae (fig. 4, A).

L'ovéjecteur comporte une première partie à parois musculeuses et une seconde à parois minces; l'ensemble mesure $680 \mu$. Les deux utérus suivent d'abord un trajet parallèle vers l'extrémité postérieure du corps, puis l'un d'eux prend une direction oppo- 


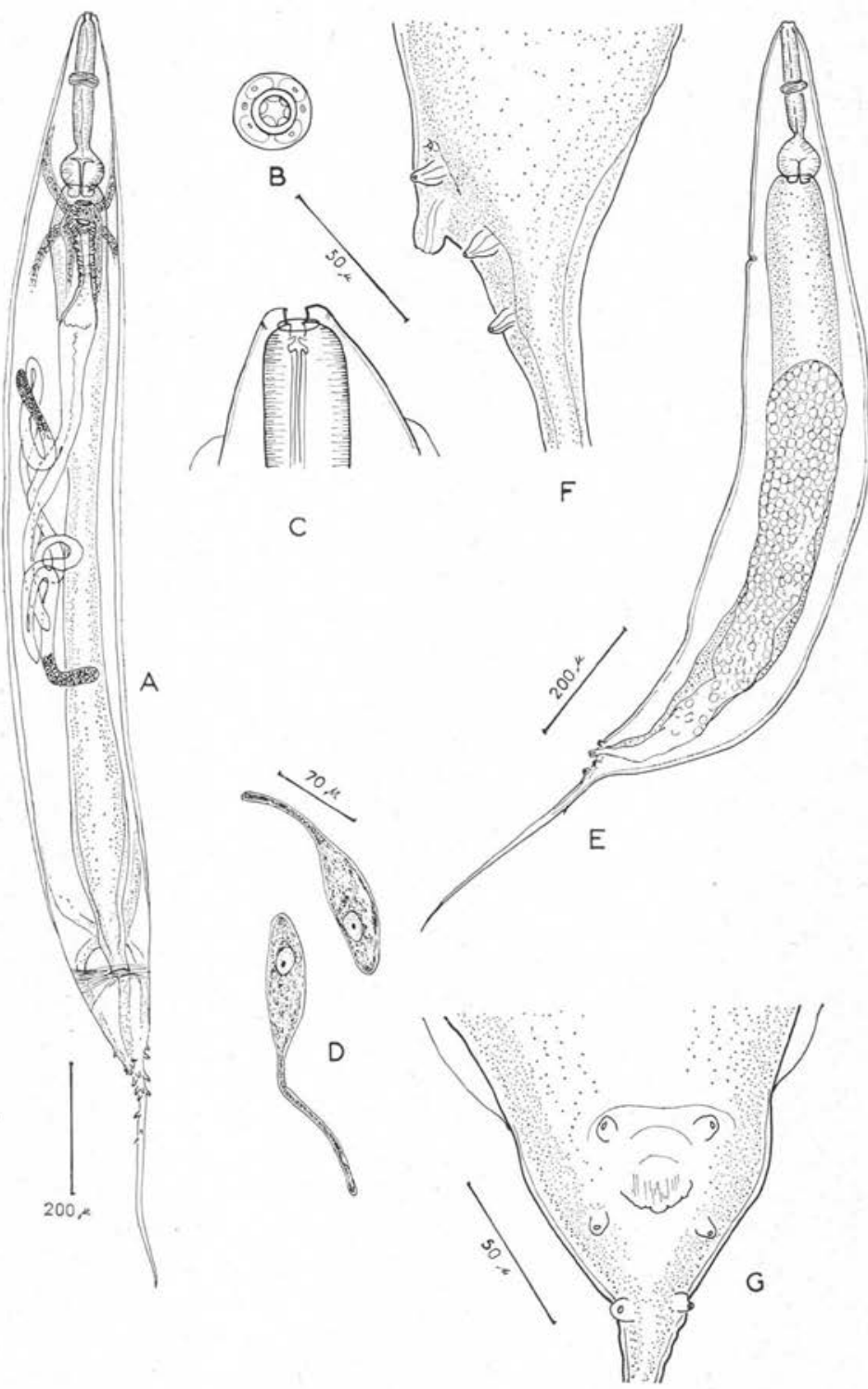

Fig. 4. - Parathelandros ovocaudatus n. sp. A, femelle, vue ventrale. B, tête de la femelle, vue apicale. C, extrémité antérieure de la femelle, vue médiane. D, œufs. E, mâle, vue latérale. F, extrémité postérieure du mâle, vue latérale. G, extrémité postérieure du mâle, vue ventrale 
sée et s'oriente vers l'extrémité antérieure. Les œufs, petits, ovales, asymétriques, portent à un de leurs pôles un filament long et épais où leur protoplasme s'introduit (fig. 4, D). La longueur des œufs, sans compter le filament, est de 100 à $110 \mu$ pour une largeur de $30 \mu$; leur longueur totale, filament compris, varie de 185 à $235 \mu$. Le corps se termine par une queue, longue de $600 \mu$, dont la partie é:roite présente, à sa naissance, une série d'épines coniques.

\section{Discussion.}

L'espèce que nous venons de décrire se distingue aisément par les œufs munis à l'un de leurs pôles d'un filament long et épais. Ce caractère n'a pas d'équivalent chez les autres espèces du genre Parathelandros Baylis, 1930.

Nous pensons que l'espèce est nouvelle et proposons pour elle le nom de Parathelandros ovocaudatus $\mathrm{n}$. $\mathrm{sp}$.

\section{5) THELANDROS MERIDIONALIS Chabaud et Brygoo, 1962 (Oxyuridae).}

Cette espèce est facile à reconnaître par la paire de papilles post-cloacales coalescentes et par l'ornementation cuticulaire chitinoïde péricloacale.

Elle a été décrite chez des Iguanidae du genre Oplurus, chez plusieurs espèces du genre Chamaeleo et chez l'Ophidien Acrantophis dumerilii Ian, à Madagascar. Nousmêmes l'avons trouvée une fois chez le Geckonidae Phelsuma mutabilis (Grandidier) (986 F) de Zampongotra, localité où précisément elle avait été signalée chez Oplurus cyclurus (Merren), et également chez divers Sauriens que nous citerons dans'les chapitres suivants.

\section{6) THELANDROS MACULATUS n. sp. (Oxyuridae)}

\section{Matériel étudié.}

Les spécimens qui portent l'étiquette $660 \mathrm{G}$ proviennent du rectum d'un Geckolepis maculata Peters, de Nossi-Bé.

\section{Description.}

Corps fusiforme, de petite taille, recouvert d'une cuticule épaisse, blanchâtre. Ailes latérales absentes.

Mâle : corps petit, de $1,55 \mathrm{~mm}$ de longueur sur $250 \mu$ de largeur. Extrémité céphalique plate. Bouche triangulaire. Deux amphides latérales; les papilles céphaliques ont été difficiles à observer. Autour du cadre buccal existe une fine ornementation cuticulaire représentée en $5 \mathrm{~F}$. L'œsophage mesure $390 \mu$ de longueur. Anneau nerveux à $50 \mu$, pore excréteur à $210 \mu$, de l'apex.

L'extrémité postérieure tronquée se termine par un appendice dorsal pointu, long de $70 \mu$. Le cloaque s'ouvre à $90 \mu$ de la pointe caudale. Ventralement, trois paires de papilles : une paire pré-cloacale, une post-cloacale située latéralement, et une paire plus petite située à la base de l'appendice caudal. Présence d'un spicu'e faiblement chitinisé long de $80 \mu$ (fig. 5, D, E). 
Femelle: corps de $3,3 \mathrm{~mm}$ de longueur sur $350 \mu$ de largeur. Extrémité antérieure étroite. Bouche hexagonale. Entre les motifs de l'ornementation cuticulaire apicale, nous avons décelé quatre petites papilles et les deux amphides latérales (fig. 5, B). CEsophage court, mesurant $680 \mu$. Anneau nerveux, pore excréteur et vulve respectivement à $140 \mu$, $700 \mu$ et $800 \mu$ de l'apex.

L'ovéjecteur, très long, se dirige vers l'extrémité postérieure du corps et, au niveau de l'intestin postérieur, il se divise en deux utérus dont les branches remontent vers la région antérieure de la femelle. Chaque utérus aboutit à un réceptacle séminal relié à l'ovaire par l'oviducte. Les ovaires, très longs, sont situés dans la région vulvaire (fig. 5, A). Les œufs, ovales, operculés, mesurent $110 \mu$ de long sur $50 \mu$ de large. Queue courte, conique, de $200 \mu$ de longueur.

Larve (fig. 5, G) : parmi les adultes, nous avons trouvé une douzaine de larves à corps allongé, de $1,47 \mathrm{~mm}$ de longueur sur $270 \mu$ de largeur, à cuticule chitineuse portant de nombreuses épines. Ces épines apparaissent au niveau de la mi-longueur de l'œsophage; elles sont alors fines et forment des rangées transversales régulières. Vers l'arrière du corps, elles augmentent de taille, mais s'espacent et sont disposées irrégulièrement. L'extrémité antérieure de la larve présente, en surface, une sorte de collerette.

L'extrémité postérieure se termine par une queue longue de $230 \mu$, brusquement effilée après l'anus, et ne portant pas d'épines cuticulaires. L'appareil digestif est semblable à celui des adultes. Esophage long de $175 \mu$.

\section{Discussion.}

Thelandros meridionalis Chabaud et Brygoo, 1962, parasite de Reptiles malgaches variés, diffère de nos exemplaires par les caractères suivants : la paire de papilles postcloacales caractéristique de $T$. meridionalis est très réduite et presque imperceptible chez nos exemplaires; l'ornementation chitinoïde péricloacale est absente chez l'espèce que nous décrivons; les ovaires ne s'enroulent pas autour de la partie postérieure de l'œsophage, ils restent situés au voisinage de la vulve sans la dépasser.

Parmi les Thelandros, notre espèce possède, comme caractères particuliers : l'extrémité postérieure du mâle (pointe longue et fine), le cône génital peu saillant, l'absence de frange cuticulaire pré-cloacale, l'existence de trois paires de papilles génitales bien développées (ia petite paire post-cloacale, très difficile à voir, semble formée de deux éléments coalescents), le dernier stade larvaire (fortement spinulé).

L'espèce qui se rapproche le plus de la nôtre, par la plupart des caractères cités ci-dessus, est $T$. californiensis Read et Amrein, 1952, parasite de Xantusia vigiles Baird et de $X$. henshanvi Stejneger, de Californie ; mais, beaucoup d'espèces sont trop mal connues pour qu'il soit possible actuellement d'établir les affinités des Thelandros entre eux.

Nous pensons donc que l'espèce est nouvelle et proposons pour elle le nom de Thelandros maculatus n. sp. 

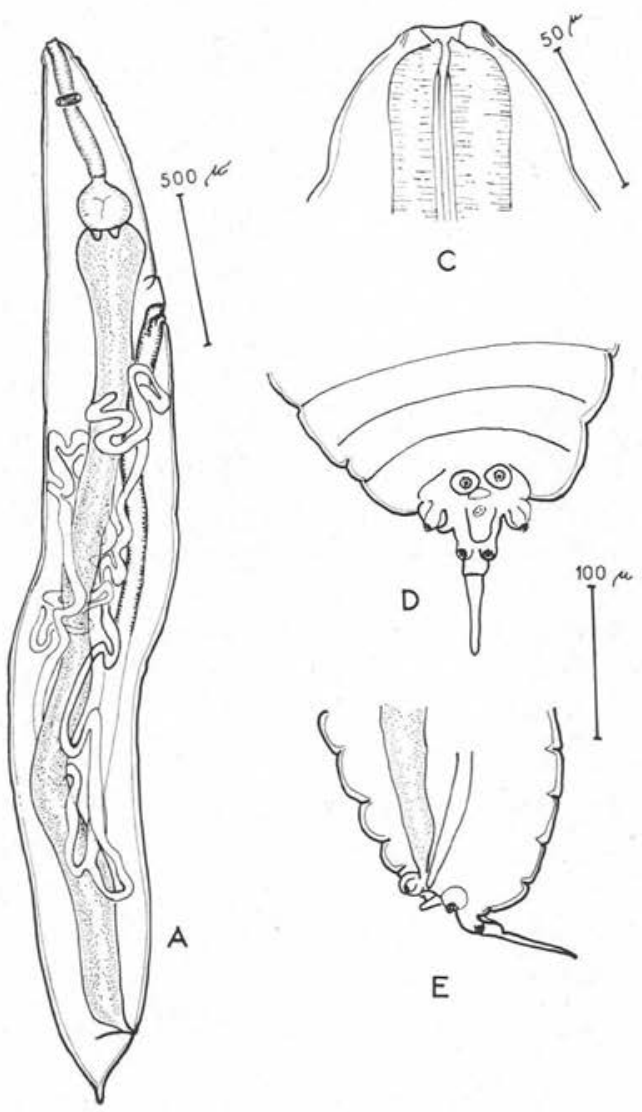

E
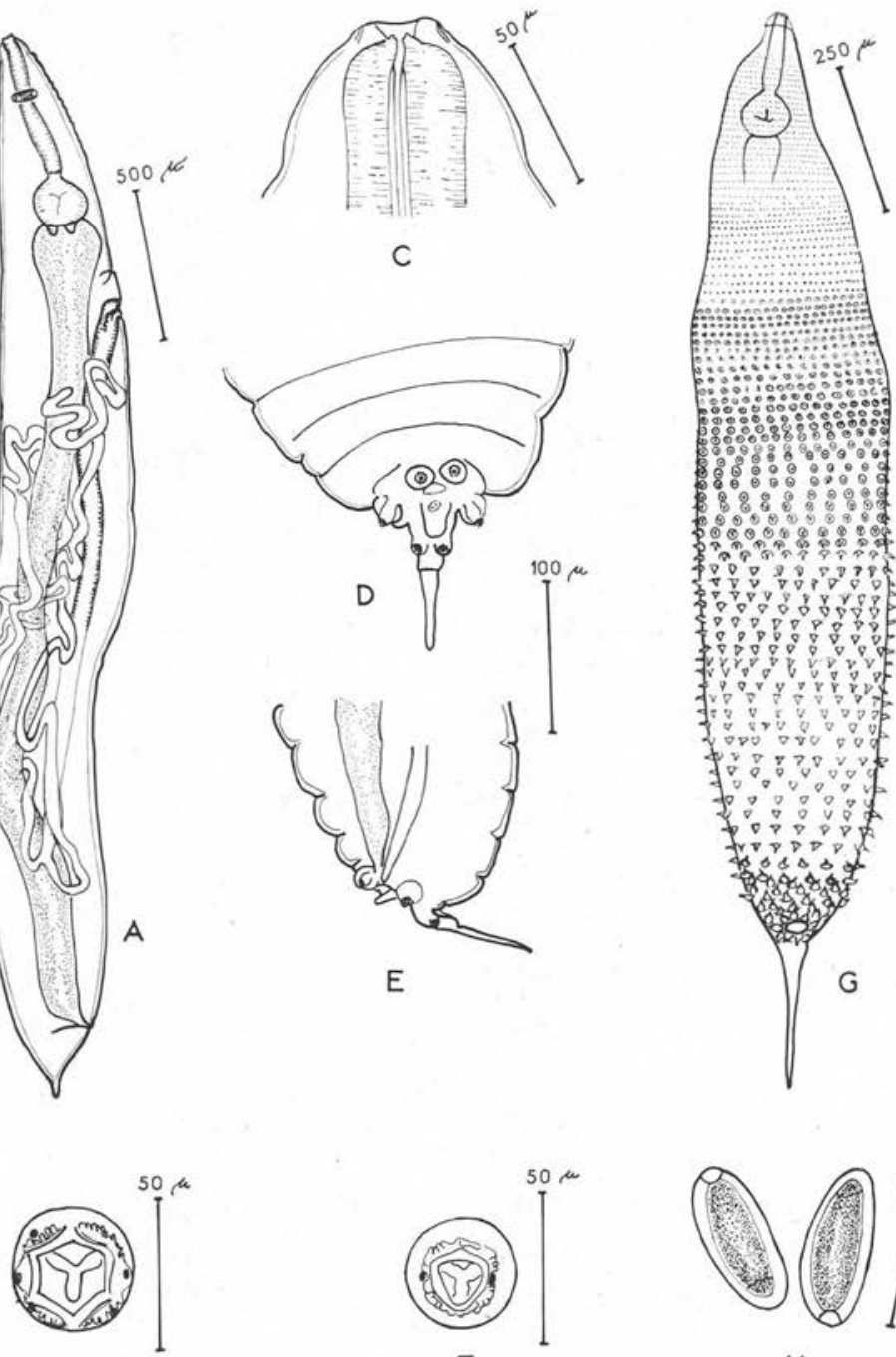

B

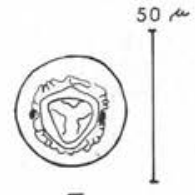

$\mathrm{F}$

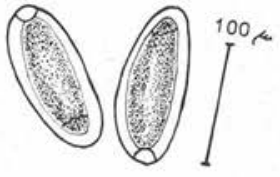

$\mathrm{H}$

Fig. 5. - Thelandros maculatus n. sp. A, femelle, vue latérale. B, tête de la femelle, vue apicale. C, extrémité antérieure du mâle, vue médiane. D, extrémité postérieure du mâle, vue ventrale. E, extrémité postérieure du mâle, vue latérale. F, tête du mâle, vue apicale. G, larve, vue ventrale. H, œufs. 
7) SPINICAUDA (MOACIRIA) FREITASI Chabaud et Brygoo, 1960 (Heterakidae).

Nous avons trouvé constamment ce parasite chez quatre Uroplatus fimbriatus (Schneider) (279 E, 988 F, $990 \mathrm{~F}, 1011 \mathrm{~F}$ ), dont nous ne connaissons pas la provenance géographique à Madagascar. Il a été tout d'abord décrit chez des Caméléons et chez trois autres Uroplatus.

8) BEFILARIA URSCHI Chabaud, Anderson et Brygoo, 1959 (Onchocercidae).

L'espèce a été décrite chez le Geckonidae Phelsuma madagascariensis Gray de Nossy-Bé ; nous l'avons retrouvée deux fois chez ce même hôte ( 659 G, 661 G), également à Amporoha (Nossy-Bé). La localisation dans l'hôte, qui était inconnue, a pu, cette fois, être précisée : les Vers vivent dans la cavité générale du Reptile.

Cette espèce appartient à un des genres endémiques à Madagascar, et se caractérise principalement par l'aspect général de l'extrémité postérieure du mâle, la situation très postérieure de la vulve, et la dilatation très marquée, en forme de disque, de la région antérieure de l'œsophage.

Cette Filaire paraît donc être très spécifique et avoir une distribution géographique très étroite.

\section{B. - Caractères de la Faune}

\section{1) Spécificité.}

La spécificité chez les Geckos est assez faible. (Voir tableau I).

D'après les données actuelles, une seule espèce de Nématodes de Geckonoidea est étroitement spécifique: la Filaire Befilaria urschi trouvée seulement chez Phelsuma madagascariensis.

Les deux Parathelandros et Pharyngodon brevibursata constituent un groupe de trois Oxyures qui ont été trouvés chez des Geckos très variés, mais non chez d'autres Reptiles.

L'Oxyuride Thelandros maculatus n'a été trouvé qu'une seule fois, chez un Gecko.

Le Seuratide Skrjabinelazia ornata et l'Heterakide Spinicauda (Moaciria) freitasi sont peu spécifiques. Nous les avons trouvés chez les autres groupes de Sauriens de Madagascar.

Thelandros meridionalis est également peu spécifique.

\section{2) Endémisme et affinités.}

Toutes les espèces sont endémiques à l'échelle spécifique, sauf la Filaire Befilaria urschi qui l'est à l'échelle générique.

Le genre Skrjabinelazia est connu de Lacertidae en Russie et en Chine.

Pharyngodon brevibursata est une espèce apparemment primitive et se rapproche des formes nord-africaines, australiennes et sud-américaines du genre. 
Parathelandros dossae paraît avoir des affinités avec plusieurs espèces connues chez des Geckos et des Mabuyas d'Afrique orientale et méridionale.

Parathelandros ovocaudatus est très particulier par la forme de ses œufs, mais se rapproche également des formes africaines.

Thelandros maculatus et $T$. meridionalis appartiennent à un genre qui est très homogène dans le monde entier, et dans lequel il est difficile d'établir des affinités.

Spinicauda (Moaciria) freitasi possède des affinités avec les formes paléarctiques et sud-américaines.

Befilaria urschi, seule espèce du genre est la forme la plus endémique rencontrée chez les Geckos. Le genre a cependant des affinités avec les genres d'Amérique du Nord Macdonaldius Khanna, 1933 et Saurofi'aria Caballero, 1954.

Il semble donc que les affinités de cette faune soient très variées: au cours de la discussion des espèces, nous avons cité l'Amérique du Sud deux fois, l'Amérique du Nord, trois fois, l'Afrique du Nord deux fois, l'Afrique orientale une fois, l'Afrique du Sud une fois, l'Afrique centrale une fois, l'Australie deux fois, l'Asie une fois, l'Europe une fois.

\section{3) Equilibre de la faune.}

Chaque espèce examinée possède un ou parfois deux Oxyures dans le rectum et accidentellement un Seuratoidea, un Heterakoidea ou un Spirurida.

Par rapport à ce qui est connu chez les Geckos des autres régions du monde, la faune des Nématodes étudiée chez 32 Geckos malgaches est donc d'une richesse moyenne et sa composition en genres n'est pas aberrante.

\section{Nématodes d'Iguanoidea}

Nous avons examiné des Nématodes provenant d'Iguanidae appartenant aux genres Chalarodon et Oplurus.

\section{A. - Etude des espèces}

1) PHARYNGODON DIMORPHA, Chabaud et Brygoo, 1962 (Oxyuridae).

L'espèce, décrite par Chabaud et Brygoo en 1962, a été récoltée dans le rectum d'un Chamaeleo pardalis Cuvier de Fort-Dauphin. A la même époque, ces auteurs ont examiné dix-sept autres $C$. pardalis et quatre $C$. lateralis Gray, de la même région et le résultat fut négatif. Plus tard, en étudiant le matériel d'un Oplurus cyclurus Merr. de Zampongotra, l'espèce a été retrouvée.

Nous-mêmes l'avons identifiée très fréquemment chez les Iguanes: trois fois sur trois chez Chalarodon madagascariensis Peters $(728 \mathrm{G}, 743 \mathrm{G}$ et $753 \mathrm{G})$ de Tuléar, neuf fois sur vingt chez Oplurus sebae (Dum. et Bibr.), (701 G, 703 G, 713 G, 714 G, $715 \mathrm{G}, 716 \mathrm{G}, 717 \mathrm{G}, 721 \mathrm{G}$ et $748 \mathrm{G}$ ) d'Ampijoroa et Antsingy, deux fois sur trois 


\begin{tabular}{|c|c|c|c|c|c|c|c|c|}
\hline & & Nombre & DE $S P$ & MENS & ROUVÉS & POR & RS D & \\
\hline $\begin{array}{c}\text { HôTes } \\
\text { Geckonoidea }\end{array}$ & 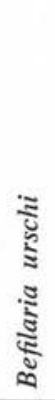 & 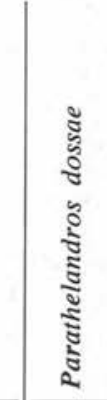 & 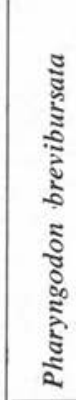 & 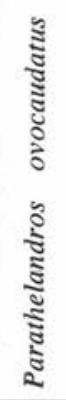 & 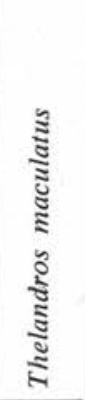 & 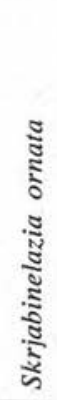 & 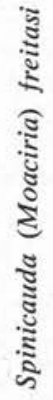 & 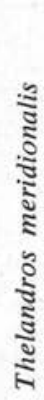 \\
\hline Geckolepis maculata ..... & & & 1 & & 1 & & & \\
\hline Geckolepis sp. ......... & & & 2 & & & & & \\
\hline Hemidactylus frenatus .... & & 1 & & & & & & \\
\hline H. mabouia .......... & & 1 & & & & & & \\
\hline Phelsuma lineatum $\ldots . .$. & & 3 & & & & 3 & & \\
\hline P. madagascariensis $\ldots \ldots$ & 2 & 2 & & & & & & \\
\hline P. mutabilis $\ldots \ldots \ldots \ldots$ & & & & 3 & & & & 1 \\
\hline Phelsuma sp. . . . . . . & & 1 & 2 & 1 & & & & \\
\hline Phyllodactylus bastardi .... & & 3 & & & & & & \\
\hline Uroplatus fimbriatus ..... & & & & & & & 7 & \\
\hline $\begin{array}{l}\text { Hôtes D'AUTRES SUPER- } \\
\text { FAMILLES } \ldots \ldots \ldots \ldots \ldots \cdots \cdots\end{array}$ & $*$ & & - & - & 一 & + & + & + \\
\hline
\end{tabular}

Tableau I. - Spécificité des Nématodes parasites de Gekonoidea. Nous signalons : a) espèces fréquentes trouvées chez une seule espèce d'hôte $\left.\left({ }^{*}\right), b\right)$ espèces trouvées uniquement dans la superfamille des Geckonoidea $(-), c$ ) espèces trouvées chez les autres superfamilles de Sauriens (+). Les chiffres indiquent le nombre d'hôtes trouvés positifs

chez $O$. cyclurus $(437 \mathrm{G}$ et $751 \mathrm{G})$ de Tuléar et d'Ambaratra et deux fois sur quatre chez $O$. fierinensis Grandidier $(736 \mathrm{G}$ et $738 \mathrm{G})$ de Tuléar.

Ainsi que l'avaient soupçonné Chabaud et Brygoo, l'espèce peut donc être considérée comme un parasite normal des Iguanes qui s'égare parfois chez des Caméléons. 


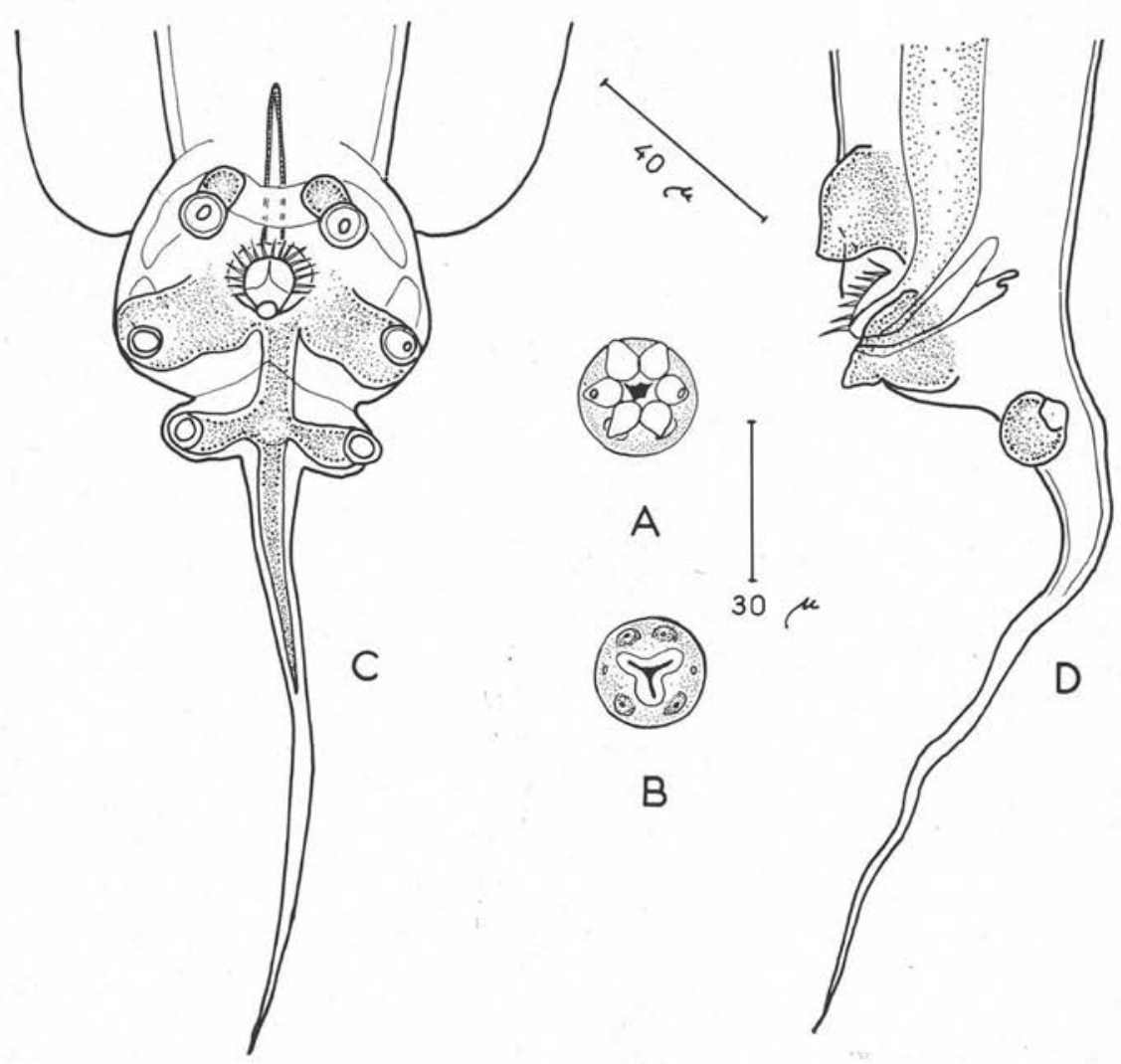

FIG. 6. - Pharyngodon dimorpha Chabaud et Brygoo, 1962. A, tête du mâle, vue apicale superficielle. B, tête du mâle, vue apicale, au niveau des papilles. $C$, extrémité postérieure du mâle, vue ventrale. D, extrémité postérieure du mâle, vue latérale

Nous donnons quelques figures pour compléter l'étude faite par Chabaud et Brygoo, notamment la tête du mâle en vue apicale (fig. 6, A, B). Les figures 6, C, D, montrent que le cône génital est orné de soies qui, sur certains exemplaires, sont bien saillantes en avant.

2) THELANDROS MERIDIONALIS Chabaud et Brygoo, 1962 (Oxyuridae).

Cette espèce, peu spécifique, déjà citée comme parasite des Geckos, a été trouvée une fois chez Oplurus sebae Dum. et Bibr. (415 F) du vivarium de Tsimbazaza et une fois chez Oplurus saxicola (Grandidier), (656 G) de Tuléar. 
FIG. 7. - Thelandros meridionalis Chabaud et Brygoo, 1962. Mâle. Extrémité postérieure

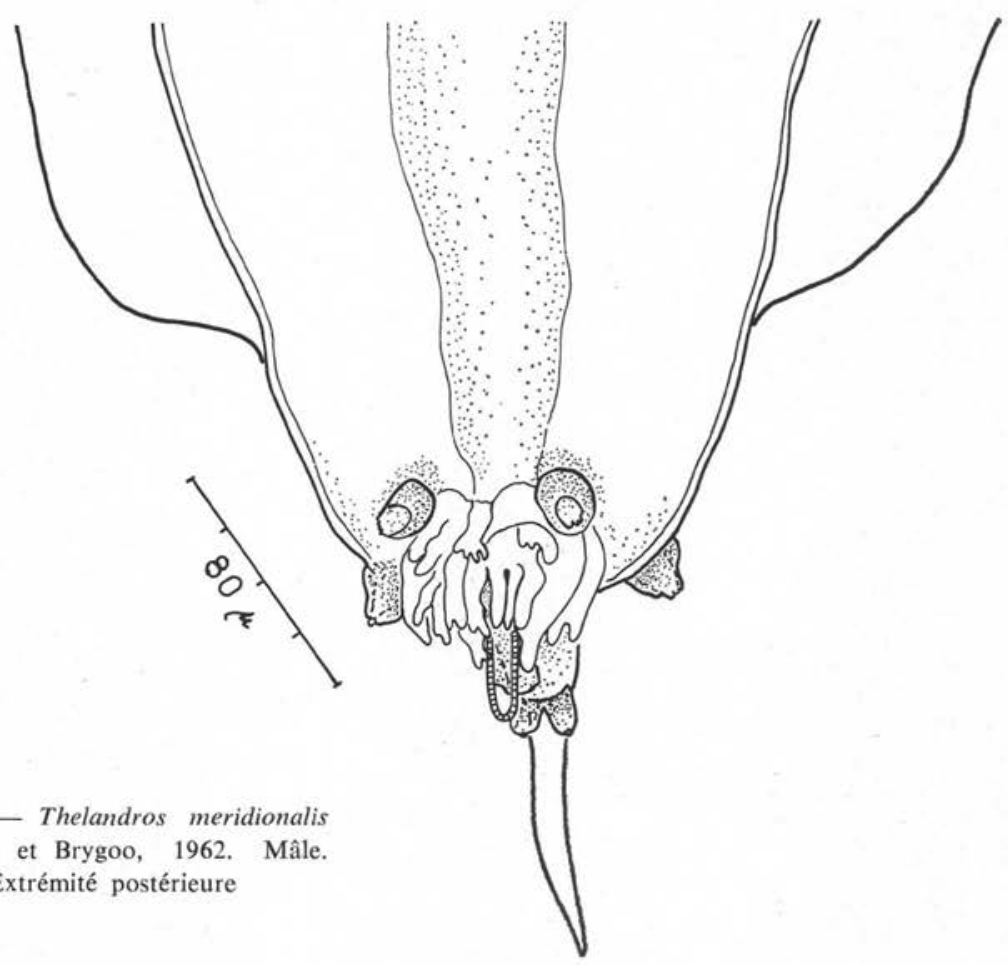

Nous figurons l'extrémité postérieure d'un mâle provenant d'un Oplurus sebae car l'ornementation péricloacale très comp.exe y apparaît particulièrement bien (fig. $7, \mathrm{~A}$ ).

3) THELANDROS BLANCHARDI n. sp. (Oxyuridae).

\section{Matériel étudié.}

Ces spécimens, qui portent l'étiquette $1035 \mathrm{~F}$, ont été recueillis dans le rectum d'un Oplurus quadrimaculatus A. Dumer., de Ranohira-Isalo, associés à T. chabaudi, espèce que nous décrirons ci-après. Nous les avons identifiés à plusieurs reprises chez la même espèce d'hôte $(1033 \mathrm{~F}, 1036 \mathrm{~F}, 1038 \mathrm{~F}, 1040 \mathrm{~F}, 741 \mathrm{G}, 772 \mathrm{G}, 774 \mathrm{G})$ tous provenant de différentes régions de l'Ile.

\section{Description.}

Mâle: corps petit et effilé de $1,54 \mathrm{~mm}$ de longueur sur $110 \mu$ de largeur. Tête petite et conique. Bouche à trois lèvres petites et peu saillantes, légèrement incisées à l'apex (fig. 8, B). En arrière des lèvres se trouve une membrane transparente qui comble partiellement les espaces intralabiaux et dépasse les lèvres en avant. Quatre reliefs arron- 
dis submédians paraissent porter chacun deux papilles. Une paire d'amphides non saillantes occupe une position latérale. Esophage musculaire terminé par un bulbe valvulé long au total de $480 \mu$. Intestin dans la région dorsale du corps. Anneau nerveux à $115 \mu$ et pore excréteur à $600 \mu$ de l'apex (fig. 8, A).

Extrémité postérieure ayant trois paires de papilles: la première paire, de taille moyenne, plus ventrale, et la seconde paire, grande et plus dorsale, sont pré-cloacales ; la troisième, petite, se trouve sur la queue. Ventralement, entre les deux premières paires de papilles et en avant du cloaque existe une très fine membrane à bord non denté (fig. $8, \mathrm{C}, \mathrm{D}$ ). Spicule de $79 \mu$ très caractérisique par sa protubérance ventrale. La paroi dorsale de sa gaine est chitinoïde et forme un étui autour du spicule, constituant ainsi une partie du gubernaculum ; celui-ci se prolonge par une branche se dirigeant vers la queue et une autre dans le cône génital saillant. Pointe caudale courte $(75 \mu)$ et mince.

Femelle: corps long de $2,96 \mathrm{~mm}$, large de $380 \mu$ au niveau du pore excréteur. Tête petite, tronquée. Bouche ample à contour circulaire, munie de six lèvres, ayant chacune une petite cavité renfermant pour les dorsa:es et ventrales une papiile, pour les latérales une amphide (fig. 9, D). En coupe optique, on voit une membrane interne, insérée sur chacun des trois lobes œsophagiens, dirigée en avant et qui obiure en partie la cavité buccale (fig. 9, E, F). Esophage musculeux long au total de $880 \mu$, terminé par un bulbe. Intestin large et peu sinueux. Aaneau nerveux et pore excréteur se trouvant respectivement à $110 \mu$ et $1,2 \mathrm{~mm}$ de l'apex.

Vulve à $820 \mu$ de l'extrémité postérieure. Ovéjecieur muscuieux de $340 \mu$ de longueur. Utérus impair long, se divisant en ceux branches. Les deux ovaires ne dépassent pas le niveau du bulbe œsophagien. Eufs de $90 \mu$ sur $50 \mu$, peu nombreux, donnant un petit embryon au moment de la ponte. Queue courte $(170 \mu)$.

En étudiant ce matériel, nous avons trouvé qu'il existe des varia:ions importantes entre les spécimens de la même espèce. Il semble, en effet, possibie de distinguer :

- Deux types chez le mâle.

En relation avec la longueur variable du corps, on note, chez les spécimens à corps long, un œsophage court et, chez les autres un peu p'us petits, un œsophage occupant presque la moitié du corps.

- Trois types chez les femelles :

a) Femelles grandes à queue courte et œsophage long. Nous donnons les dimensions d'un spécimen de ce type, qui correspond à celui décrit plus haut (fig. 9, A) :

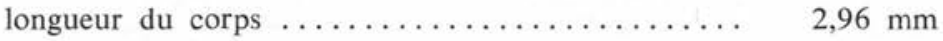
longueur de la queue ................. $170 \mu$
longueur de l'œsophage $\ldots \ldots \ldots \ldots \ldots \ldots \ldots \ldots \quad 880 \mu$

b) Femelles moyennes à queue et œsophage moyens (fig. 9, B). Dimensions d'un spécimen de ce type :

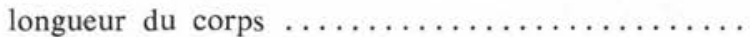

$2,48 \mathrm{~mm}$

longueur de la queue .................. $190 \mu$

longueur de l'œsophage $\ldots \ldots \ldots \ldots \ldots \ldots \ldots \ldots \ldots$ 


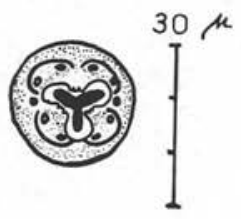

B
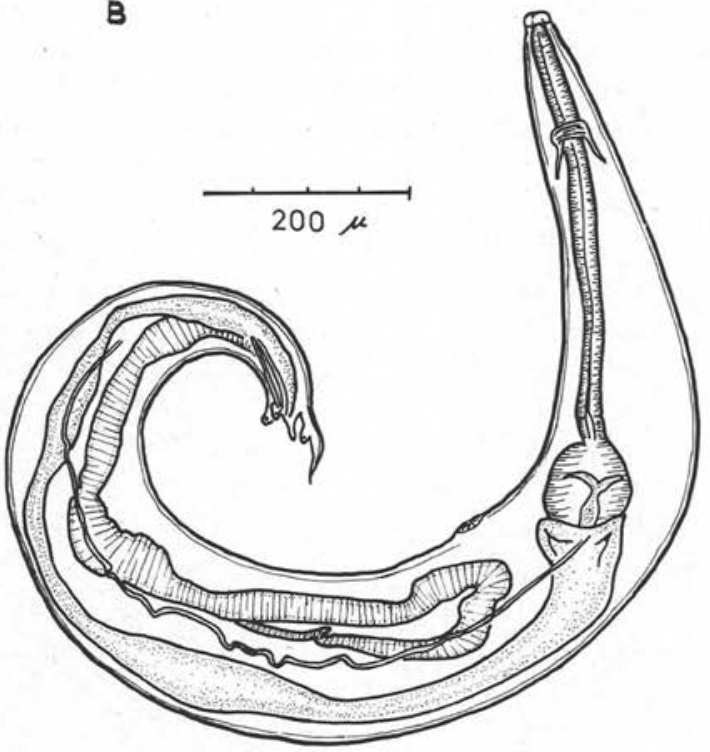

A

Fig. 8. - Thelandros blanchardi n. sp. Mâle. A, vue latérale. B, tête, vue apicale. C, extrémité postérieure, vue ventrale. $\mathrm{D}$, extrémité postérieure, vue latérale
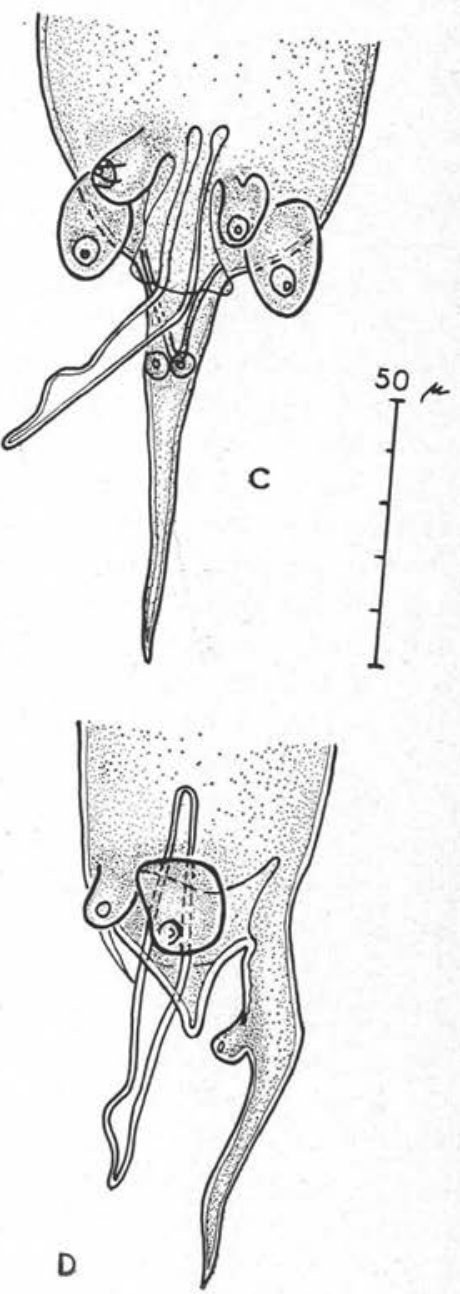

c) Femelles petites à queue longue et œsophage court (fig. 9, C). Dimensions d'un spécimen de ce type :

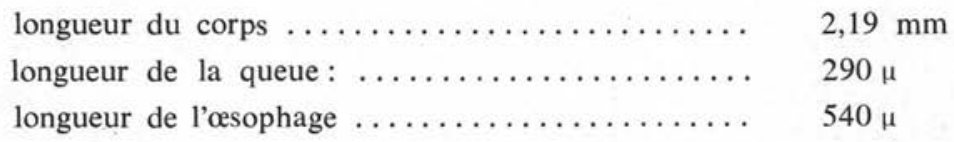

En raison des variations, nous avons désigné comme holotype un mâle à corps long et œsophage court. 


\section{Discussion.}

Bien que ces parasites aient tous les caractères du genre Thelandros Wedl, 1862, ils présentent certaines particularités qui permettent de les différencier des autres espèces déjà décrites. Ces caractéristiques sont : le parasitisme chez un Iguane, la forme du spicule, la forme de la tête de la femelle.

L'espèce est remarquablement proche de Thelandros versterae Petter, 1966, parasite de Testudo tentoria verreauxi en Afrique du Sud. Les seules différences facilement appréciables sont la position plus antérieure de la vulve et le bord denticulé de la membrane pré-cloacale des parasites de la Tortue. En outre, A. Petter a constaté, elle aussi, l'existence de trois types morphologiques qu'elle a individualisés en sous-espèces: $T$. versterae versterae, $T$. versterae weilleae, $T$. versterae tcheprakovae.

Cet étroit parallélisme entre l'espèce parasite d'une Tortue sud-africaine et celle d'un Iguane malgache est difficile à expliquer, d'autant plus que ce dernier à un régime insectivore. Quoi qu'il en soit, nous considérons l'espèce comme nouvelle et la nommons Thelandros blanchardi, n. sp., en souvenir de Raphaël Blanchard, l'un des fondadeurs de la parasitologie contemporaine.

\section{4) THELANDROS CHABAUDI, n. sp. (Oxyuridae).}

Les spécimens mâles et femelles, qui portent l'étiquette $1035 \mathrm{~F}$, ont été récoltés dans le rectum d'un Oplurus quadrimaculatus A. Dumer., à Ranohira-Isalo. Le même Nématode a été retrouvé chez neuf Iguanes de même espèce dans la même région.

\section{Description.}

Mâle: corps petit et effilé, mesurant 1,62 mm sur $130 \mu$. Extrémité antérieure étroite. Tête conique et petite (fig. 10, C). En vue apicale, quatre petites papilles submédianes et deux amphides latérales (fig. 10, B). Bouche à trois lèvres communiquant avec un œsophage long, étroit, musculeux, formant bulbe à son extrémité, long de $650 \mu$ (bulbe compris). Anneau nerveux et pore excréteur se trouvant respectivement à $85 \mu$ et $690 \mu$ de l'extrémité antérieure (fig. 10, A).

Extrémité postérieure portant trois paires de papilles : la première paire pré-anale et la seconde ad-anale, toutes deux de même taille, la troisième plus petite et se trouvant sur la queue. Ventralement, entre les papilles de la première paire, existe une petite membrane dont l'apex est très découpé (fig. 10, D). Gubernaculum en $\mathrm{V}$, mesurant $25 \mu$. Spicule fin et transparent, de $55 \mu$ de long. Queue courte et mesurant $50 \mu$ (fig. 10, $\mathrm{E}, \mathrm{F})$.

Femelle: beaucoup plus grande que le mâle. Corps étroit, long de 1,9 mm sur $190 \mu$ de large au niveau du pore excréteur (fig. 11, A). Tête conique présentant, en vue apicale, quatre papilles submédianes, plus six reliefs légèrement saillants, correspondant peut-être à six papil'es internes. Deux amphides latérales (fig. 11, B). La bouche, à trois lèvres courtes et épaisses, se trouve obturée par une membrane chitinoïde saillante en avant (fig. 11, C, D, E, F), dont la constitution est visible sur coupe optique, et qui prend naissance à l'intérieur du lobe labial un peu en arrière de la partie apicale 


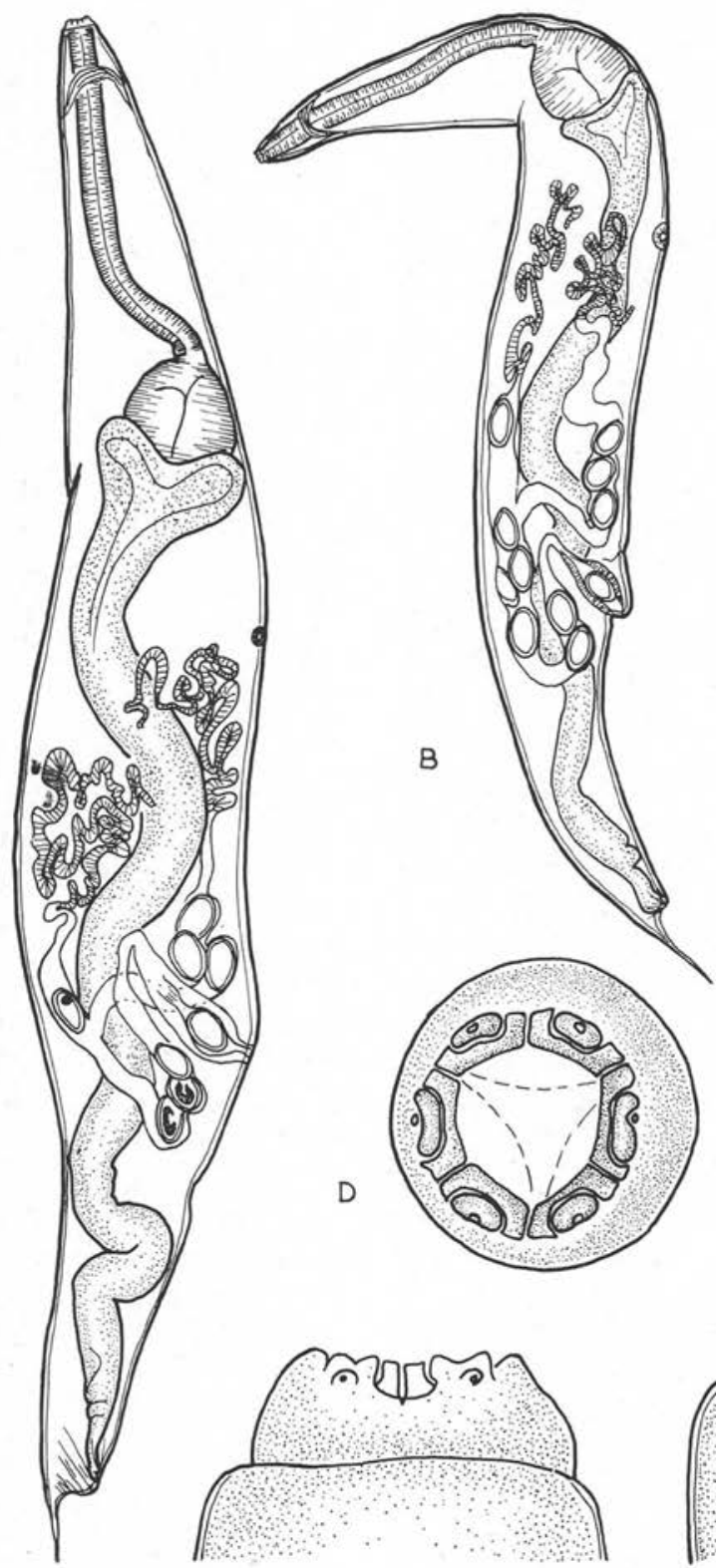

A
E
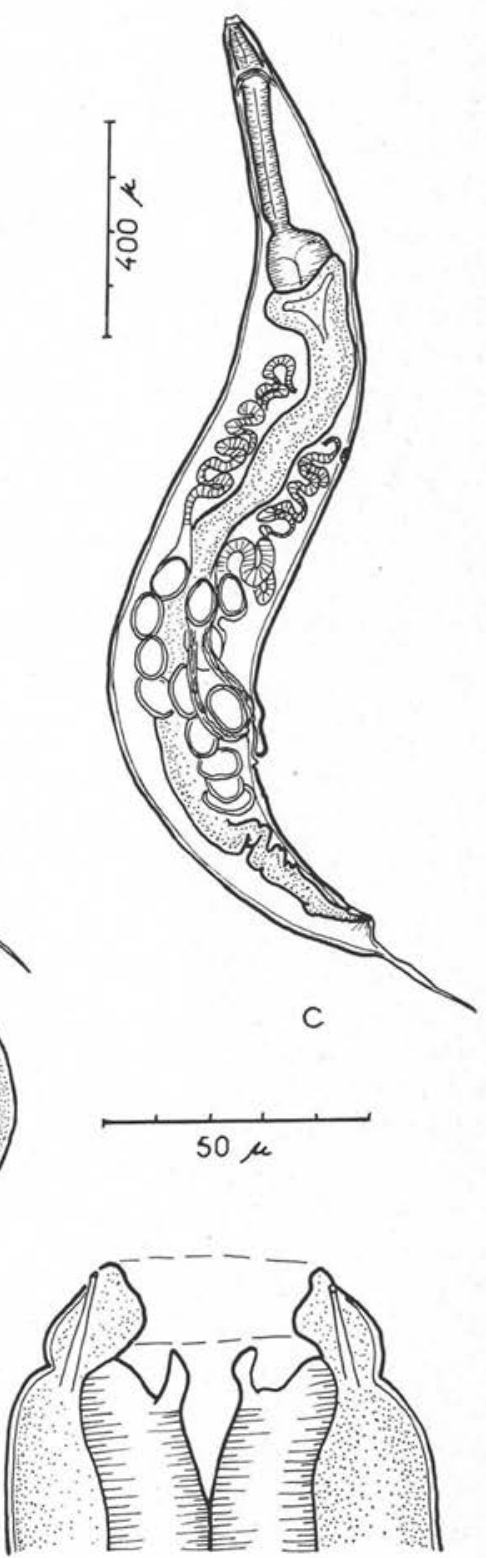

F

FIG. 9. - Thelandros blanchardi n. sp. Femelle. A, B, C, vues latérales. D, tête, vue apicale. E, extrémité antérieure, vue ventrale superficielle. F, extrémité antérieure, vue médiane 


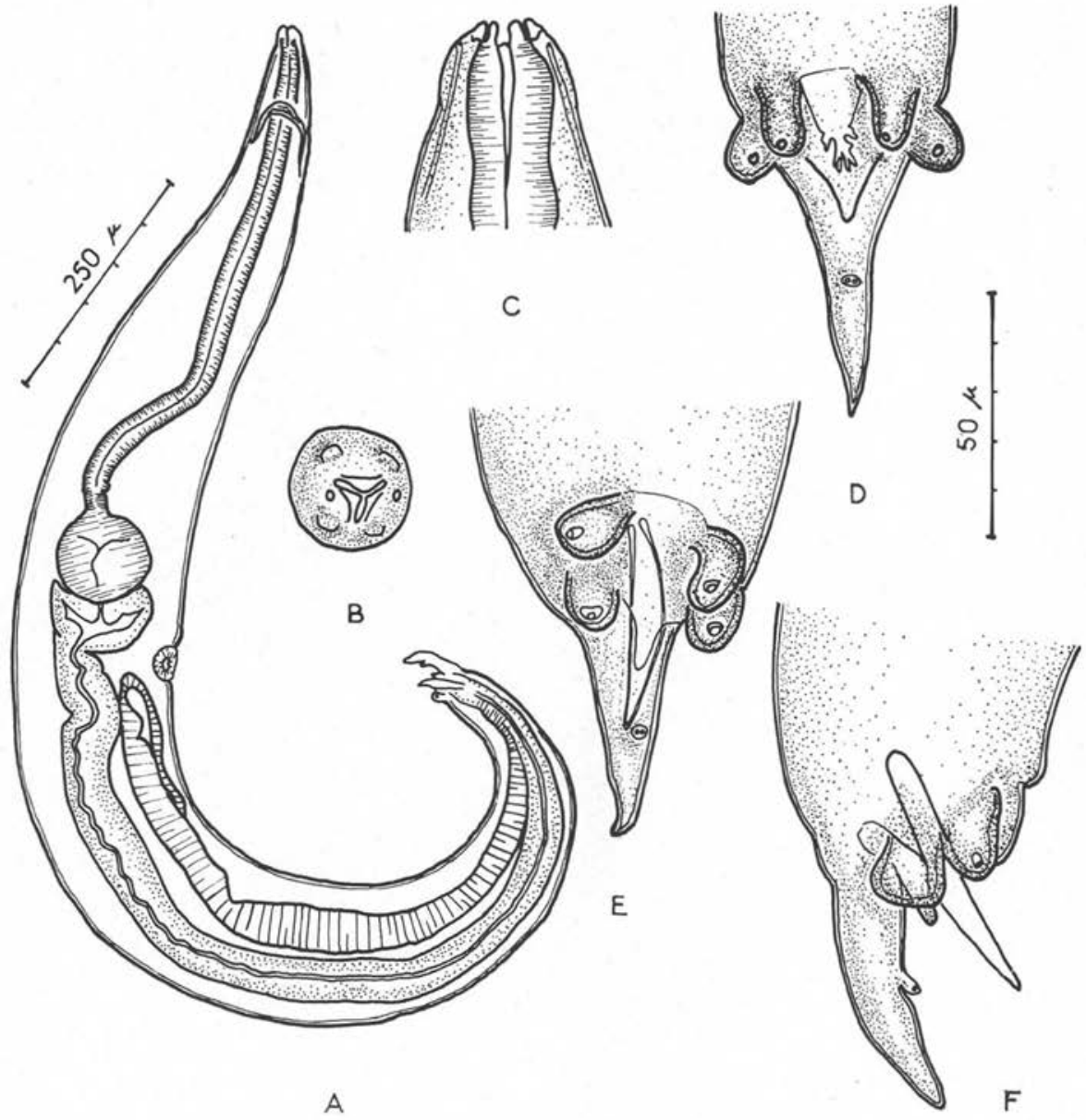

Fig. 10. - Thelandros chabaudi n. sp. Mâle. A, vue latérale. B, tête, vue apicale. C, extrémité antérieure, vue ventrale. D, extrémité postérieure, vue ventrale au niveau de l'ornementation péricloacale. E, extrémité postérieure, vue ventrale au niveau du spicule. F, extrémité postérieure, vue latérale

de la lèvre. Bouche communiquant avec une petite capsule buccale et une grande cavité œsophagienne. Attachées au fond de cette cavité, se trouvent trois structures coniques en forme de baguettes, libres dans toute leur longueur, et s'élevant jusqu'au niveau de la bouche (fig. 11, G). L'œesophage, long et musculeux, mesure $650 \mu$, bulbe compris. Anneau nerveux et pore excréteur respectivement à $150 \mu$ et $750 \mu$ de l'extrémité antérieure du corps.

L'appareil reproducteur est constitué par deux ovaires enroulés autour de l'intestin et se prolongeant chacun par un utérus. L'utérus impair est très long. L'ovéjecteur à 


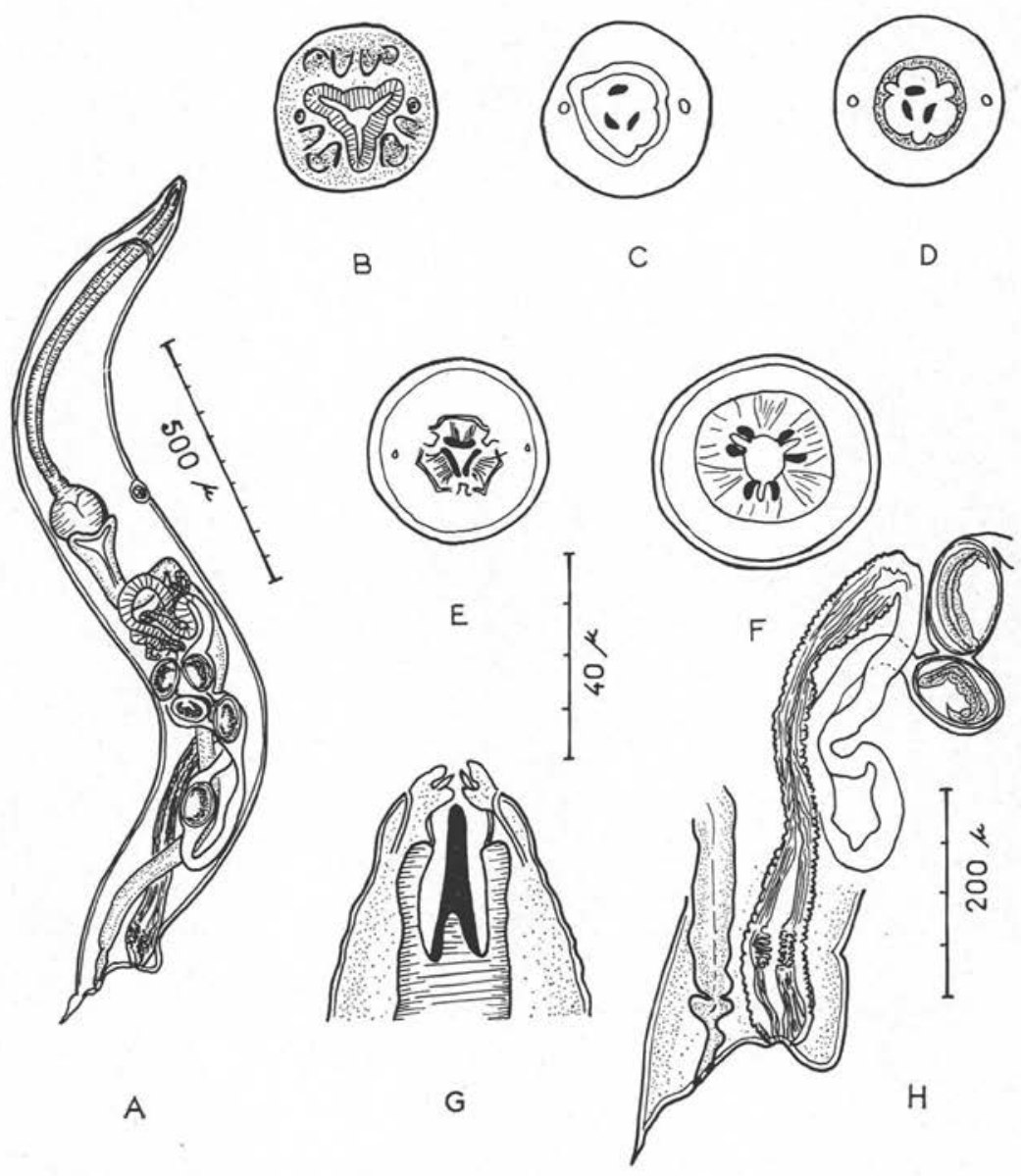

FIG. 11. - Thelandros chabaudi n. sp. Femelle. A, vue latérale. B, tête, vue apicale superficielle. C, D, tête, vues apicales au niveau des baguettes pharyngiennes. E, tête, vue apicale au niveau du fond de la capsule buccale. F, tête, vue apicale au niveau du début de l'œsophage. G, extrémité antérieure, vue médiane. $\mathrm{H}$, ovéjecteur

parois musculeuses, long de $525 \mu$, présente près de la vulve un petit sphincter (fig. 11, H). Vulve avec lèvre antérieure saillante, s'ouvrant à $160 \mu$ de l'extrémité postérieure du corps. L'utérus contient des œufs de $110 \mu$ sur $70 \mu$ renfermant un embryon. Queue courte $(60 \mu)$, et mince.

\section{Discussion.}

L'espèce appartient au genre The'andros Weld, 1862, par la forme, la disposition et le nombre de papilles caudales. 
Elle s'apparente beaucoup à Thelandros alatus Wedl, 1862, parasite de l'Agamidae Uromastix spinipes (Daudin) d'Afrique du Nord, mais en diffère par la structure très caractéristique de la cavité buccaie et par la position de la vulve s'ouvrant à l'extrémité postérieure.

Nous pensons donc que l'espèce malgache est nouvelle et la désignons sous le nom de Thelandros chabaudi n. sp., en témoignage de notre pronfonde gratitude envers le Professeur A. G. Chabaud.

5) SPINICAUDA (MOACIRIA) FREITASI Chabaud et Brygoo, 1960 (Heterakidae).

Trouvée fréquemment chez les Chamaeleo et chez les Uroplatus, cette espèce a été identifiée chez les Iguanes Op urus quadrimaculatus Gray et $O$. fierinensis Grandidire de Tuléar, et également chez les Gerrhosauridae et chez les Scincidae.

\section{6) STRONGYLURIS WINTERI n. sp. (Heterakidae).}

\section{Matériel étudié.}

Le matériel de cette espìce est constitué de six mâles et de trois femelles, qui portent l'étiquette $576 \mathrm{G}$, provenant du rectum d'un Oplurus grandidieri Mocquard, de Ihosy.

\section{Description.}

Le corps de ces Nématodes est effilé, recouvert d'une cuticu'e épaisse, pourvu d'ailes latérales qui naissent presque au niveau de l'anneau nerveux et se prolongent jusqu'à l'extrémité postérieure. De nombreuses papilles somatiques pédonculées sont disposées dans la région céphalique et œsophagienne. La tête, de forme conique, est séparée du corps par un rétrécissem.ent bien défini. La bouche, petite, est bordée de trois lèvres dont les bords latéraux se terminent par une aile fine et transparente qui couvre l'espace interlabial (fig. 12, C). En vue apicale, la coupe optique de ces ailes labiales donne l'impression qu'il existe des crochets ou baguettes sur toute la longueur de l'espace interlabial (fig. 12, C). Quatre petites papilles constituent le cycle externe et six le cycle interne. Deux amphides sont placées au milieu des lèvres latéro-ventrales. Faisant suite à la bouche, une cavité buccale est limitée par les trois lobes pharyngiens dont l'apex est denticulé et qui portent chacun une petite dent sur leur face externe, fig. 12, B).

Mâle: corps de $12 \mathrm{~mm}$ de longueur sur $350 \mu$ de largeur. Extrémité antérieure étroite. Tête de $330 \mu$ de longueur sur $450 \mu$ de largeur (ailes labiales comprises). Pharynx long de $260 \mu$. Esophage long de $1,9 \mathrm{~mm}$ (bulbe compris). Anneau nerveux et pore excréteur respectivement à $430 \mu$ et $1,35 \mathrm{~mm}$ de l'apex (fig. $12, \mathrm{~A}$ ).

Extrémité postérieure pourvue d'une bourse caudale presque circulaire de $350 \mu$ de long sur $360 \mu$ de large ; celle-ci est soutenue par 8 paires de papilles pédonculées dont : 4 pré-anales, 2 ad-anales et 2 post-anales. En outre, près du cloaque se trouvent 3 paires de papilles non pédonculées dont : 1 paire ad-anale et 2 post-anales. Entre la seconde et 


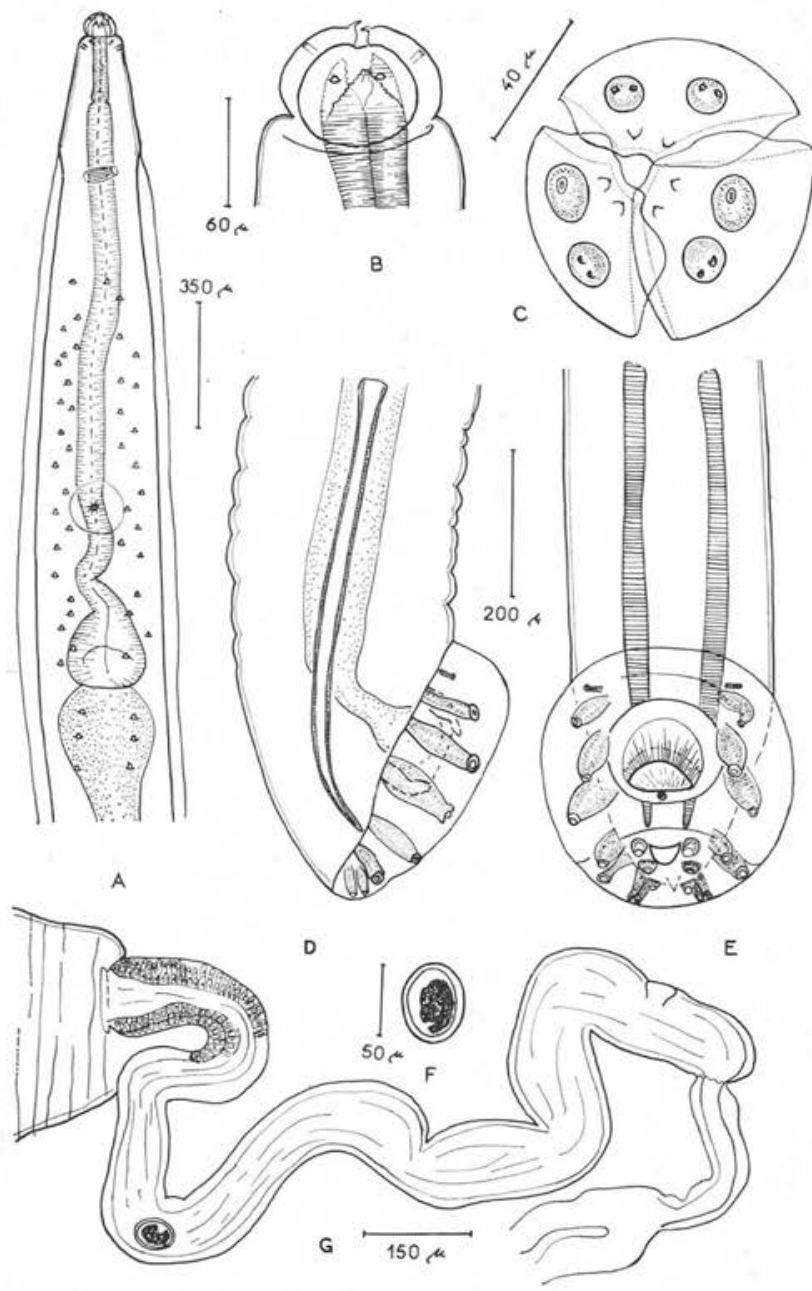

FIG. 12. - Strongyluris winteri n. sp. A, extrémité antérieure du mâle, vue ventrale. B, tête du mâle, vue dorsale, C, tête du mâle, vue apicale. D, extrémité postérieure du mâle, vue latérale. E, extrémité postérieure du mâle, vue ventrale. F, œufs. G, ovéjecteur

la quatrième paire de papilles pédonculées et au-dessus du cloaque est située une grande ventouse musculeuse de $140 \mu$ de diamètre longitudinal sur $160 \mu$ de diamètre transversal, soutenue en profondeur par un anneau chitinoïde ; l'ouverture de cette ventouse est de $40 \mu$ de longueur sur $90 \mu$ de largeur et se trouve à $120 \mu$ en avant du cloaque ; sur le bord postérieur de la ventouse est située une petite papille. Les 2 spicules sont aigus, égaux et longs de $680 \mu$. Gubernaculum absent. Queue très petite (fig. 12, D, E). 
Femelle : légèrement plus grande que le mâle, elle mesure $15 \mathrm{~mm}$ de long sur $400 \mu$ de large. Pharynx long de $450 \mu$. Esophage de $1,9 \mathrm{~mm}$ (bulbe compris). Anneau nerveux et pore excréteur respectivement à $510 \mu$ et $1,9 \mathrm{~mm}$ de l'extrémité antérieure du corps.

Vulve située à $3,4 \mathrm{~mm}$ de l'extrémité postérieure du corps. L'ovéjecteur, long de $2,125 \mathrm{~mm}$, comporte un segment di'até long de $1,8 \mathrm{~mm}$, puis un segment étroit long de $250 \mu$. Les deux branches utérines sont remplies d'œufs jaunâtres, longs de $60 \mu$ et larges de $45 \mu$ (fig. 12, F, G). Extrémité postérieure arrondie.

\section{Discussion.}

Le parasite décrit ci-dessus appartient au genre Strongyluris Mueller, 1894.

Les espèces qui constituent ce genre sont très homogènes et généralement très difficiles à distinguer les unes des autres. Les caractères morphologiques qui nous ont semblé les plus importants sont : chez le mâle, la ventouse (forme, taille, distance ventousecloaque et distance ventouse-extrémité postérieure du corps); les papilles caudales, bien qu'elles soient sujettes à des anomalies ou des malformations (disparition de certaines papilles et développement démesuré de la papille opposée ou bien totale disparition d'une paire d'entre elles) ; la position du cloaque par rapport à l'extrémité postérieure du corps ; les spicules ; l'appendice caudal. Chez la femelle, la forme de l'extrémité postérieure et la taille des œufs.

Par la forme arrondie de la bourse caudale, les espèces qui nous paraissent les plus proches sont : Strongyluris brevicaudata Mueller, 1894, S. elegans (Gendre, 1909) et $S$. chamaeleonis Baylis et Daubney, 1922.

- Strongyluris brevicaudata, Mueller, 1894, parasite d'Agama colonorum Daudin, d'Afrique occidentale, de Chamaeleo fisheri multituberculatus Nieden, $C$. fisheri Reichenow, $C$. matsetrice et $C$. dilepis dilepis (Leach), d'Afrique, et de C. tempeli (Tornier), de Tanganyika (Afrique orientale), a une ventouse plus petite et des spicules plus longs $(1,1 \mathrm{~mm})$; la pointe caudale est presque imperceptible.

- S. elegans (Gendre, 1909), parasite de Chamaeleo gracilis Hallowel, de Guinée, se distingue par l'ornementation réticulée des spicules; la ventouse est plus petite ; chez la femelle, la queue est droite, conique et pointue ; les œufs mesurent de $69 \mu$ à $98 \mu$ de longueur, sur $48 \mu$ à $58 \mu$ de largeur, et sont donc beaucoup plus grands.

- S. chamaeleonis Baylis et Daubney, 1922, parasite de Chamaeleo vulgaris Daudin, de l'Inde, se distingue aisément par l'absence d'ailes latérales et de papilles somatiques, et par l'existence de 9 paires de papilles caudales.

En raison de ces différences, nous pensons que cette espèce est nouvelle et la nommons Strongyluris winteri n. sp. à la mémoire de notre regretté collègue helminthologiste Howard A. Winter.

7) STRONGYLURIS SKRJABINI n. sp. (Heterakidae).

\section{Matériel étudié.}

Le matériel dont nous disposons porte l'étiquette $738 \mathrm{G}$ et comprend 8 mâles et 


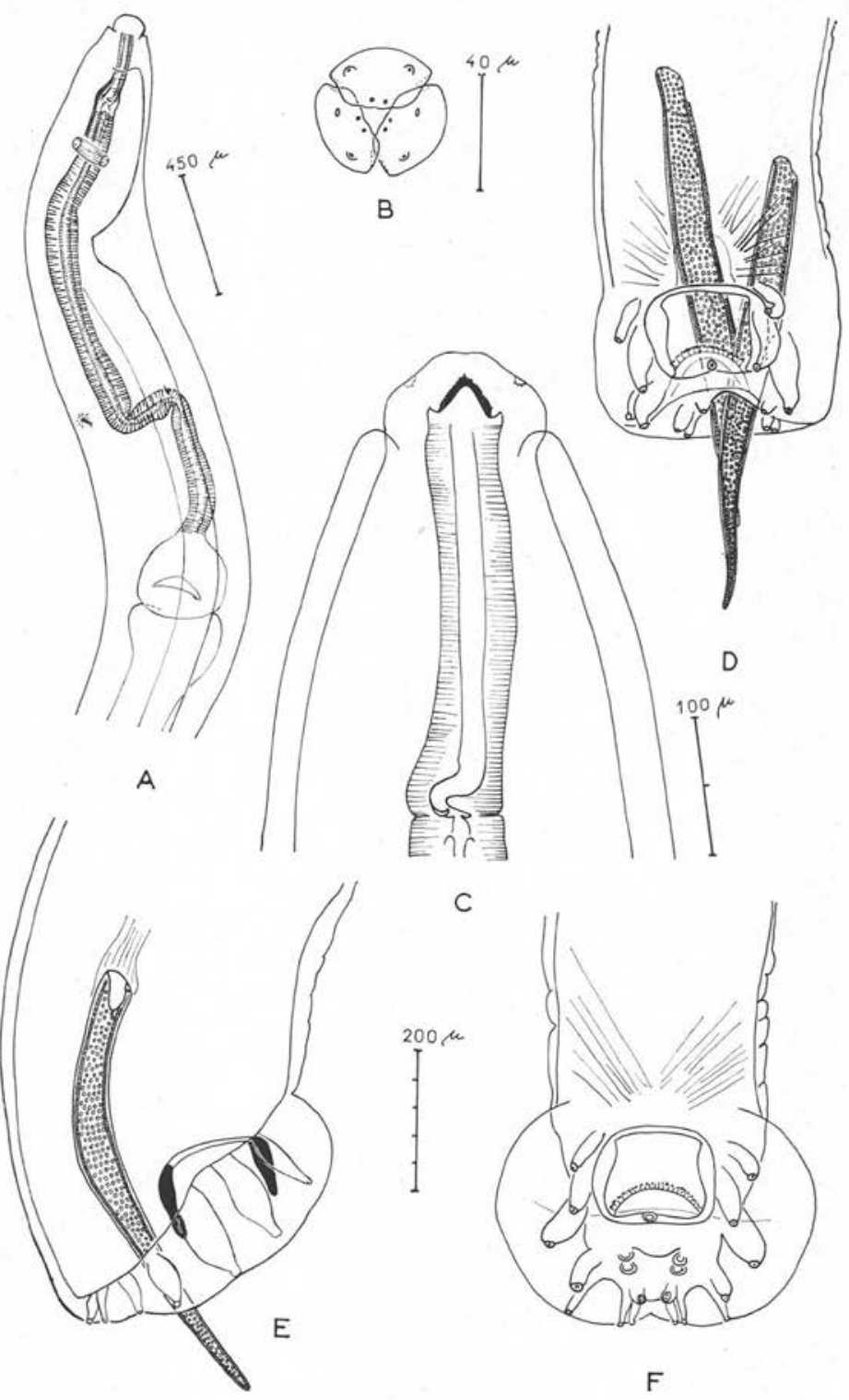

FIG. 13. - Strongyluris skrjabini n. sp. Mâle. A, extrémité antérieure, vue latérale. B, tête, vue apicale. C, extrémité antérieure, vue dorsale. D, F, extrémité postérieure, vues ventrales. E, extrémité postérieure, vue latérale 
8 femelles recueillis dans le rectum de l'Iguane Oplurus fierinensis Grandidier, de Tuléar. Nous l'avons retrouvé chez un $O$. saxicola (Grandidier) (747 G) de Tsihombé.

\section{Description.}

Chez tous les spécimens femelles, la région antérieure est contractée et constitue un segment à cuticule plissée, suivi d'un étranglement très marqué au niveau duquel naissent les ailes latérales (fig. 13, G). Cette particularité n'existe pas chez les mâles. Ailes latérales courtes, prenant naissance au niveau de la partie moyenne du pharynx.

Mâle : le corps est long de $10,5 \mathrm{~mm}$ sur $510 \mu$ de largeur au niveau du bulbe œsophagien. A $200 \mu$ de l'extrémité antérieure prennent naissance les ailes latérales étroites qui se poursuivent tout le long du corps. La tête (fig. 13, B) est formée de trois lèvres égales; le bord latéral de chacune se prolonge par une aile céphalique qui se superpose à celle de la lèvre voisine; le cycle interne est formé de deux petites papilles sensorielles réfringentes placées à l'apex de chaque lèvre ; le cycle externe, de quatre papilles céphaliques peu saillantes et de deux amphides latérales. Une petite cavité buccale, haute de $50 \mu$, est limitée par les trois lobes pharyngiens à bords chitineux et denticulés. Le pharynx, long de $280 \mu$ et large de $50 \mu$, est tapissé de cuticule. En avant de l'œsophage, la lumière pharyngienne présente une courbure nettement accentuée (fig. 13, C). L'œsophage tubulaire et musculeux se termine par un bulbe piriforme; le corpus est long de $1,85 \mathrm{~mm}$ sur $75 \mu$ de large, le bulbe est long de $320 \mu$ sur $300 \mu$ de large ; l'œsophage mesure donc au total $2,17 \mathrm{~mm}$ (fig. 13, A). L'intestin, un peu élargi à son début, se rétrécit ensuite, puis conserve la même largeur jusqu'au cloaque. Anneau nerveux, diérides et pore excréteur respectivement à $510 \mu, 1,40 \mathrm{~mm}$ et $1,46 \mathrm{~mm}$ de l'apex.

L'extrémité postérieure, tronquée, sans appendice caudal, plus étroite que le reste du corps, est ornée d'une bourse caudale carrée chez un de nos exemplaires, mais ovale dans le sens transversal chez quelques autres spécimens; ventralement, elle porte une grande ventouse de $150 \mu$ de longueur sur $220 \mu$ de largeur. Le cloaque est situé entre 40 et $60 \mu$ du bord postérieur de la ventouse, soit à $80 \mu$ de l'extrémité postérieure du corps. Les larges ailes caudales sont soutenues par sept paires de papilles pédonculées: les trois premières paires, situées sur les côtés de la ventouse, augmentent de taille progressivement de la première à la troisième paire ; plus postérieures et situées sur les bords latéraux du corps, quatre paires de papilles digitiformes sont rangées par groupes de deux. Il existe également des papilles médianes: le bord postérieur de la ventouse porte une petite papille impaire, et trois paires de papilles sont post-cloacales (les deux premières étant sessiles, la troisième paire étant légèrement pédonculée et située à la base de la dernière paire digitée) (fig. 13, D, E, F). Les deux spicules égaux sont longs de $820 \mu$ et larges de $70 \mu$; leur extrémité distale se termine en pointe fine ; leur surface, criblée de fines ponctuations, a un aspect caractéristique.

Femelle: le corps, long de $12 \mathrm{~mm}$, est large de $770 \mu$ au niveau des diérides (fig. 14, A). L'extrémité céphalique, semblable à celle du mâle, mesure $100 \mu$ de long sur $110 \mu$ de large. La cavité buccale est longue de $50 \mu$. Pharynx musculeux long de $300 \mu$ et large de $60 \mu$. La courbure pharyngée pré-œsophagienne est moins accentuée que chez le mâle. L'œsophage claviforme mesure $2,74 \mathrm{~mm}$ de long sur $100 \mu$ de large. L'anneau 
nerveux, les diérides et le pore excréteur sont situés respectivement à $650 \mu, 1,52 \mathrm{~mm}$ et $1,52 \mathrm{~mm}$ de l'apex.

La vulve se trouve à $8,49 \mathrm{~mm}$ de l'extrémité antérieure du corps (soit aux $2 / 3$ antérieurs). Les utérus gravides contiennent de nombreux œufs, ovales, longs de $80 \mu$ sur $60 \mu$ de large (fig. 14, B). L'extrémité postérieure se termine par une queue ovale longue de $310 \mu$ et large de $400 \mu$ au niveau de l'anus (fig. 14, C). La distance des phasmides à l'extrémité postérieure est de $90 \mu$.

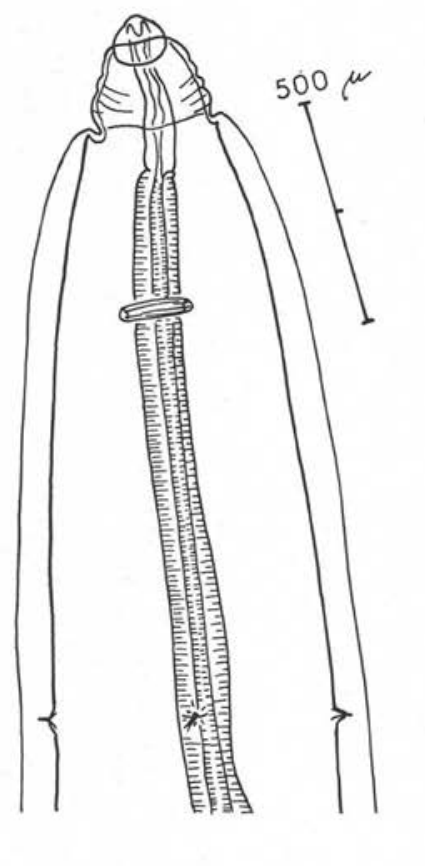

A
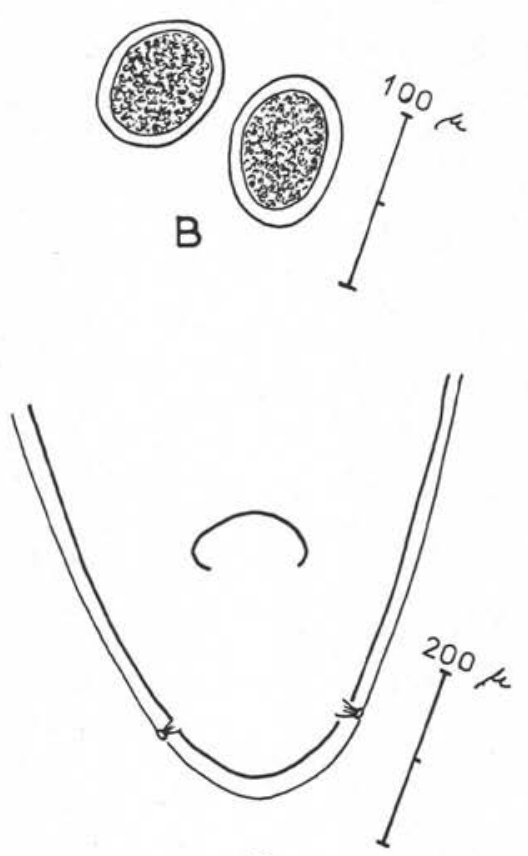

C

FIG. 14. - Strongyluris skrjabini n. sp. Femelle. A, extrémité antérieure, vue ventrale. B, œufs. C, extrémité postérieure, vue ventrale

\section{Discussion.}

Nous avons rapproché nos exemplaires de Strongyluris chamaeleonis Baylis et Daubney, 1922, de S. oscari Travassos, 1923 et de S. winteri décrit plus haut.

- S. chamaeleonis Baylis et Daubney, 1922, parasite de Chamaeleo vulgaris Daudin, de l'Inde, est différent par les caractères suivants : la taille de la ventouse est beaucoup plus petite par rapport à la bourse caudale ; les bords latéraux de la ventouse ne portent pas les trois paires de papilles pré-cloacales; la distance ventouse-extrémité postérieure du corps est plus grande; les spicules sont de taille moyenne $(1,1 \mathrm{~mm})$ 
et fins; il y a neuf paires de papilles génitales; les ailes latérales sont absentes et l'appendice caudal présent.

- S. oscari Travassos, 1923, parasite de l'Iguanidae Tropidurus sp. du Brésil, est différent de nos spécimens par les caractères suivants : la ventouse est plus petite, il y a huit paires de papilles génitales; la queue de la femelle est conique et pointue ; les œufs sont petits.

- S. winteri, parasite de l'Iguanidae Oplurus grandidieri Mocquard, de Ihosy, Madagascar, est différent par les caractères suivants : bourse caudale circulaire ; spicules minces et sans ornementation spéciale ; onze paires de papilles génitales ; appendice caudal très court.

Nous considérons notre espèce comme nouvelle et la nommons Strongyluris skrjabini $\mathrm{n}$. sp. en témoignage de respectueuse admiration pour le $\mathrm{P}^{\mathrm{r}} \mathrm{K}$. I. Skrjabin.

8) PHYSALOPTEROIDES DOLLFUSI n. sp. (Physalopteridae).

\section{Matériel étudié.}

Le matériel étudié se compose, d'une part, d'un mâle et d'une femelle qui portent l'étiquette 746 G, récolté dans l'estomac d'un Oplurus sebae (Dum. et Bibr.), d'Antsingy ; d'autre part, nous avons retrouvé des femelles provenant de l'estomac et de l'intestin antérieur du même hôte, mais originaire d'Ampijoroa. Nous avons assimilé ces femelles à l'espèce décrite ci-dessous.

\section{Description.}

Les parasites de petite taille sont grêles et revêtus d'une cuticule finement striée transversalement. Pas d'ailes latérales. L'extrémité céphalique légèrement arrondie présente deux pseudo-lèvres, chacune d'elles portant une paire de papilles submédianes et deux amphides latérales. La pseudo-lèvre droite, moins large que la gauche, est pourvue de trois dents pointues (fig. 16, C, E) ; la lèvre gauche, par contre, est plus large et légèrement saillante (fig. 16, D). Le bord buccal est lisse et non denté. Les commissures des lèvres se rabattent vers la gauche.

Mâle: corps long de $11,4 \mathrm{~mm}$ et large de $310 \mu$. La bouche s'ouvre dans une cavité buccale à parois chitinoïdes de $32 \mu$ de longueur (fig. 15, B). L'œesophage, long de $2,28 \mathrm{~mm}$, est formé de deux parties: l'une musculaire de $230 \mu$ et l'autre glandulaire de 2,05 $\mathrm{mm}$ de longueur. Anneau nerveux, diérides et pore excréteur respectivement à $210 \mu, 275 \mu$ et $165 \mu$ de l'extrémité céphalique (fig. 15, A).

Extrémité postérieure effilée, pourvue de deux ailes caudales asymétriques, dont la droite mesure $830 \mu$, et la gauche $750 \mu$ (fig. 15, E). Les papilles, petites et faiblement pédonculées, se confondent facilement avec l'ornementation cuticulaire caudale formée d'un ensemble de granulations chitinoïdes irrégulières. Douze papilles du côté droit et treize du côté gauche se disposent de la façon suivante : 8 pré-cloacales avec trois paires pédonculées latérales et une paire plus petite, sessile, située sur le bord antérieur du cloaque ; 13 post-cloacales, avec six paires sessiles latérales, plus une papille du côté gauche, faisant partie d'une septième paire probable, et deux petites paires situées 

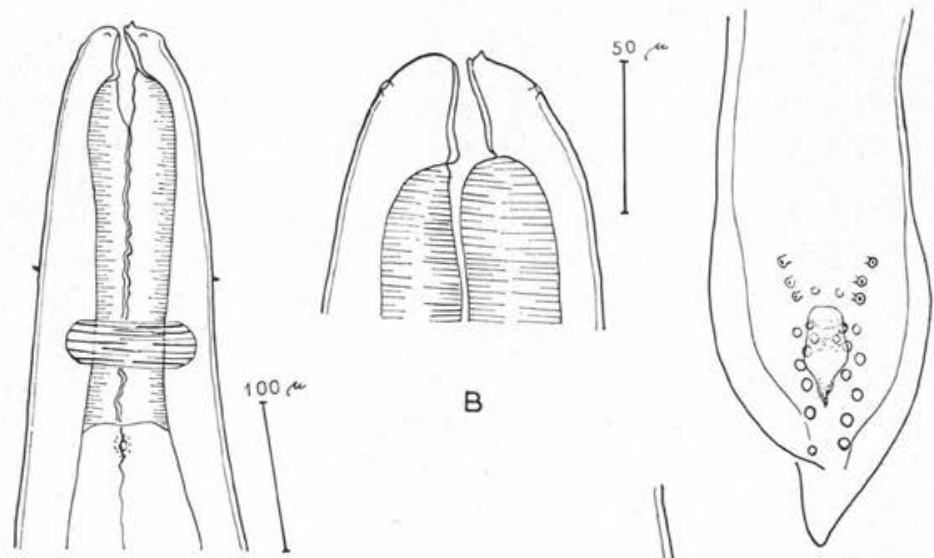

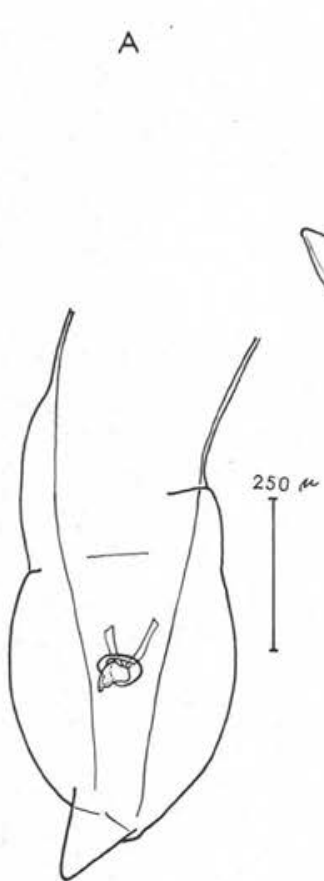

E

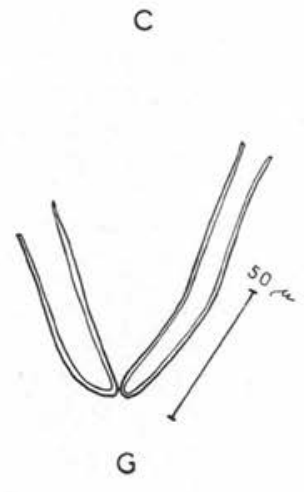

G 

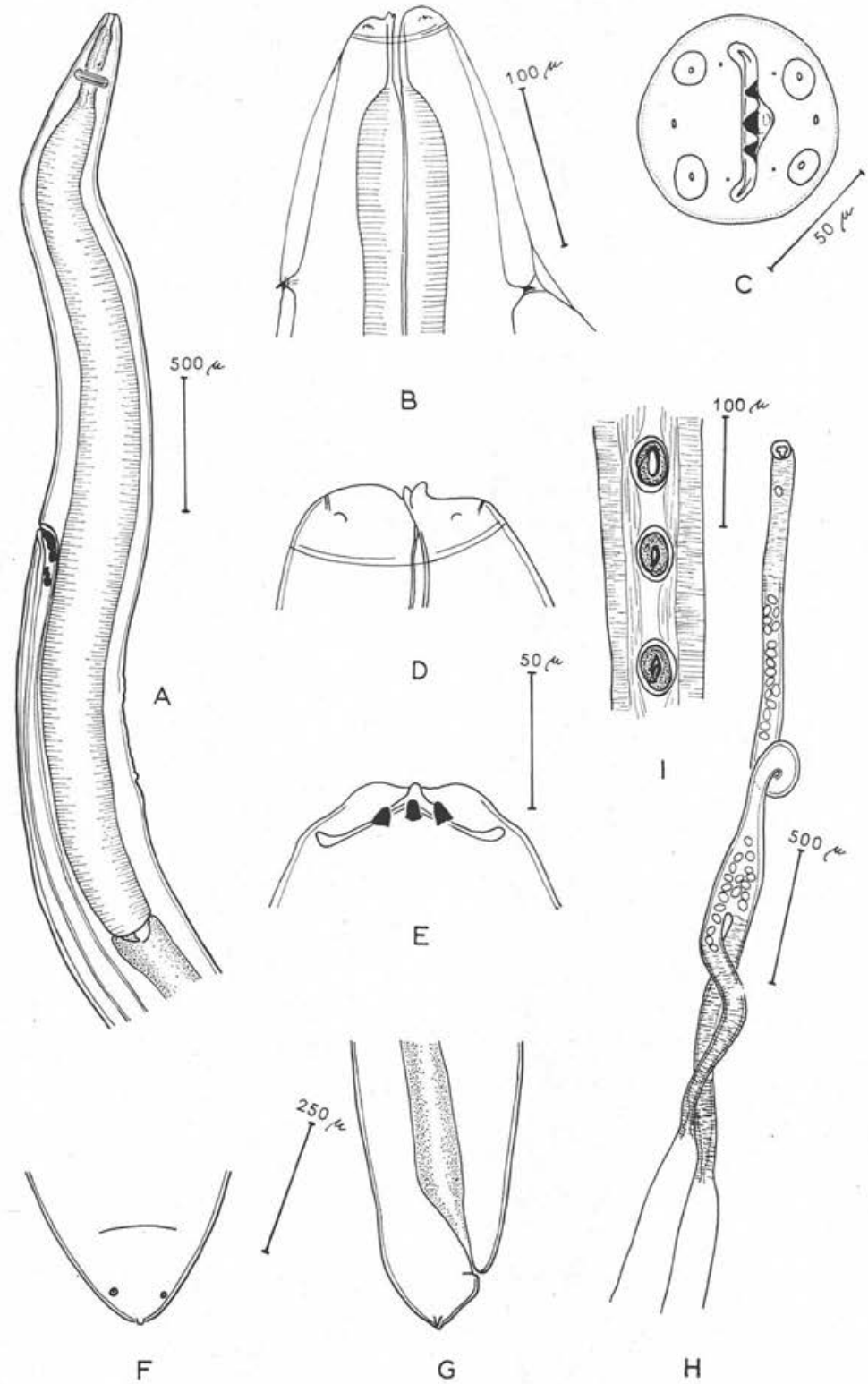

$\mathrm{H}$

Fig. 16. - Physalopteroides dollfusi n. sp. Femelle. A, extrémité antérieure, vue latérale. B, extrémité antérieure, vue ventrale. C, tête, vue apicale. D, pseudo-lèvres, vue dorsale. E, pseudo-lèvre droite, coupe optique. $\mathrm{F}$, extrémité postérieure, vue ventrale. G, extrémité postérieure, vue latérale. $\mathrm{H}$, ovéjecteur. I, œufs 
autour du cloaque (fig. 15, F). Du bord antérieur du cloaque se détache une languette membraneuse qui recouvre le cloaque et se prolonge jusqu'à l'avant-dernière paire de papilles génitales (fig. 15, C, D). Spicules subégaux, petits et peu chitinisés, le droit long de $65 \mu$, le gauche de $92 \mu$. Gubernaculum absent. Queue longue de $360 \mu$.

Femelle : corps long de $18 \mathrm{~mm}$ et large de $530 \mu$. Cavité buccale longue de $50 \mu$. Esophage long de $3,62 \mathrm{~mm}$, différencié en deux régions : la région musculaire, mesurant $320 \mu$, et la glandulaire, 3,3 mm (fig. 16, A, B). L'anneau nerveux, les diérides et le pore excréteur sont respectivement situés à $250 \mu, 200 \mu$ et $500 \mu$ de l'extrémité antérieure.

La vulve s'ouvre à $2,1 \mathrm{~mm}$ de l'apex. l'ovéjecteur (fig. 16, H), long de $3 \mathrm{~mm}$, s'élargit légèrement avant sa division en deux trompes. Les utérus constituent de véritables sacs bourrés d'œufs. Oviductes et ovaires se trouvent enroulés près du rectum. Les œufs, petits, embryonnés, de cou'eur jaunâtre, mesurent $41 \mu$ de longueur sur $25 \mu$ de largeur (fig. 16, I). La queue, courte, conique, longue de $180 \mu$, se termine par un petit mucron. Les deux phasmides se situent à $60 \mu$ de l'extrémité postérieure (fig. 16, F, G).

\section{Discussion.}

Le genre Physalopteroides Wu et Liu, 1940, est caractérisé par une armature buccale asymétrique, placée tantôt sur la lèvre droite, tantôt sur la lèvre gauche. Parmi les formes connues, quatre ont, comme notre espèce, la pseudo-lèvre droite dentée : $P$. impar (Malan, 1939) ; P. dryophisi Wu et Liu, 1940 ; P. venancioi (Lent, Freitas et Proença, 1946) ; P. chamaeleonis Chabaud et Brygoo, 1960.

Ces espèces diffèrent de la nôtre par les caractères suivants :

- P. impar (Malan, 1939), parasite de Cordylus cordylus (L.) (= Zonurus cordylus) et d'Agama atra Daudin, d'Afrique du Sud, possède cinq paires de papilles postcloacales et les papilles cervicales se situent au niveau de l'anneau nerveux.

- P. dryophisi Wu et Liu, 1940, parasite de l'Ophidien Dryophis prasinus Boyé, en Chine, possède une bourse caudale ornée de six paires de papilles pré-cloacales et quatre paires post-cloacales.

- P. venancioi (Lent, Freitas et Proença, 1946), parasite d'Amphibiens du genre Bufo du Paraguay, a une queue plus courte pourvue de quatre paires de papilles postanales.

- P. chamaeleonis Chabaud et Brygoo, 1960, parasite de Chamaeleo verrucosus Cuvier et $C$. oustaleti Mocquard, de Madagascar, est l'espèce la plus proche, mais la dentition céphalique est différente (une paire de dents sur la lèvre gauche) et le cloaque est dépourvu de la formation membraneuse décrite ci-dessus.

Nous considérons l'espèce comme nouvelle et la nommons Physalopteroides dollfusi n. sp., en témoignage d'admiration pour l'œuvre helminthologique de M. R.-Ph. Dollfus. 
9) ABBREVIATA BRYGOOI n. sp. (Physalopteridae).

\section{Matériel étudié.}

Le matériel étudié porte l'étiquette $1032 \mathrm{~F}$ et a été récolté dans l'estomac d'un Iguane, Oplurus quadrimaculatus A. Dumer, à Ranohira Isalo.
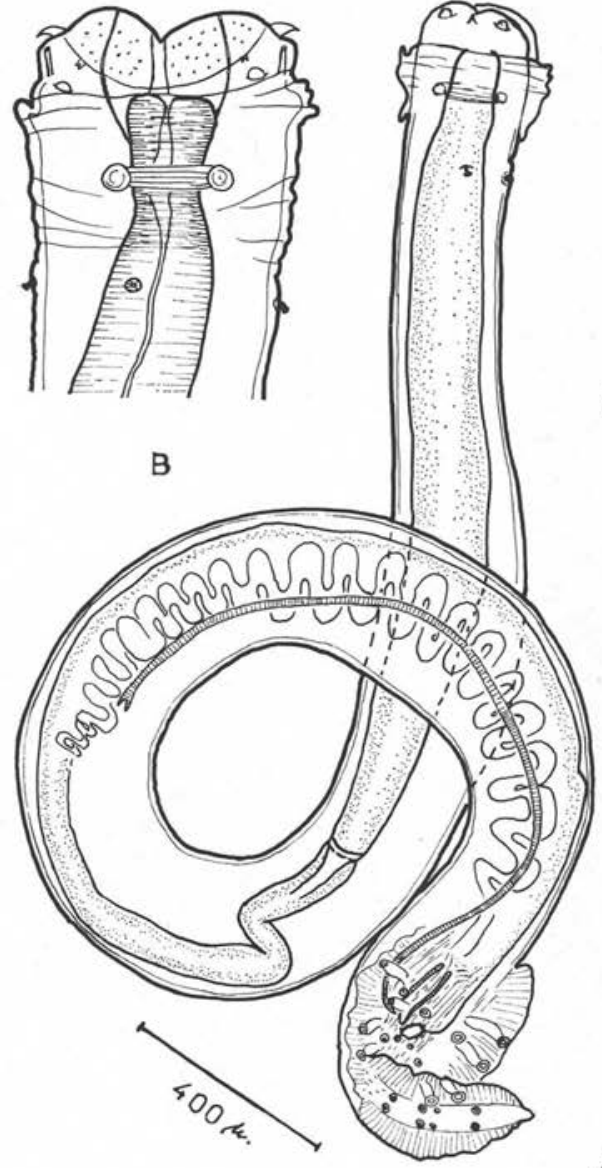

A

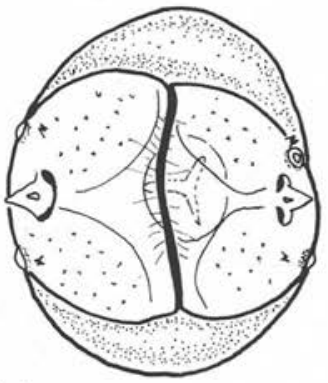

C

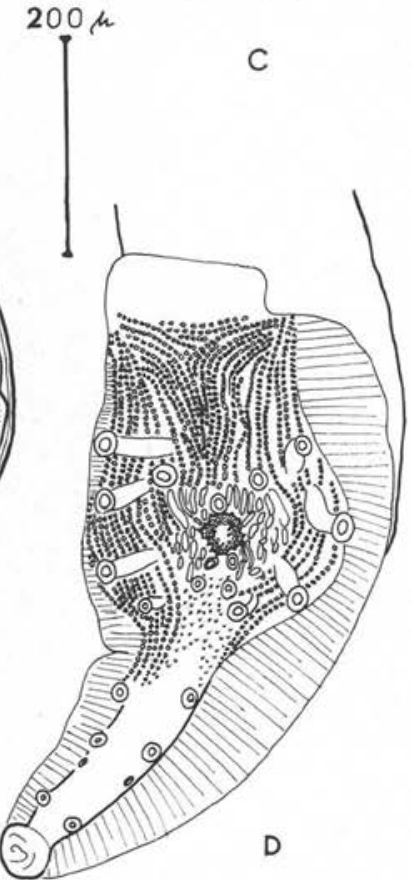

Fig. 17. - Abbreviata brygooi n. sp. A, mâle B, extrémité antérieure du mâle, vue ventrale. C, tête de la femelle, vue apicale. D, extrémité postérieure du mâle, vue ventrale

\section{Deseription.}

Mâle : corps petit, mince et transparent, mesurant $4,56 \mathrm{~mm}$ de long sur $250 \mu$ de large. Sur tous les exemplaires, l'extrémité céphalique a la structure normale d'un Abbre- 
viata, mais son aspect est inhabituel. La partie interne des pseudo-lèvres est dilatée et fait saillie en avant, si bien que les lignes où se trouvent insérées les dents ne sont plus situées sur l'axe ventro-dorsal, mais sont déportées en arrière et latéralement, comme si la bouche était largement ouverte (fig. 17, B). Extrémité antérieure de $230 \mu$ de largeur ; la cuticule se soulève pour former une collerette. Bouche à deux pseudo-lèvres
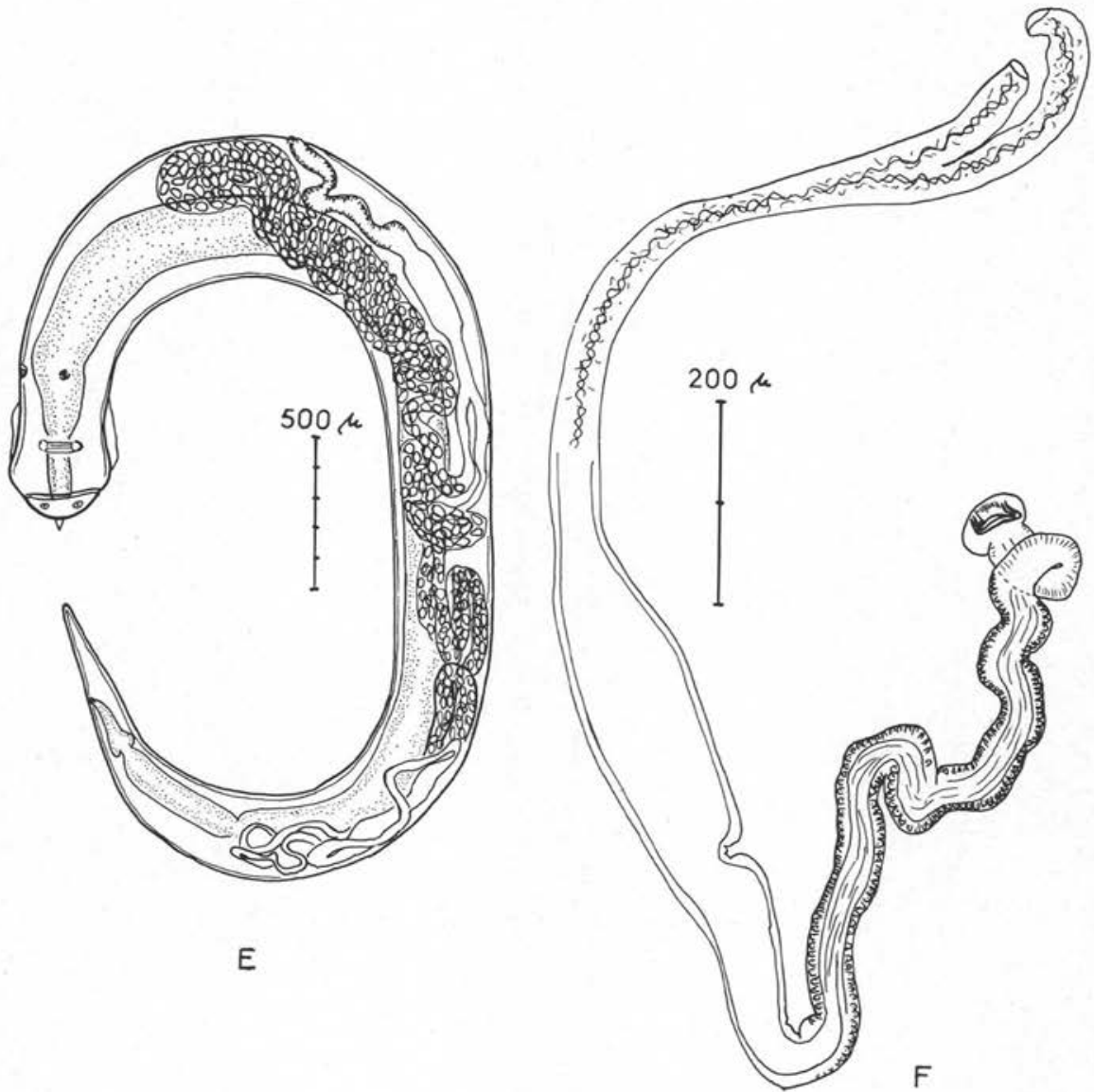

FIG. $17^{\text {bis, }}-$ Abbreviata brygooi $\mathrm{n}$. sp. E, femelle, vue latérale. F, ovéjecteur

latérales, portant chacune une grosse dent externo-latérale et une petite dent internolatérale; de chaque côté de la grosse dent se trouvent une double dent submédiane et, plus en arrière, une petite papille; entre elles, sur l'axe latéral, une amphide (fig. 17, A, B, C). Esophage de 1,6 mm de longueur totale, dont une portion musculaire antérieure de $140 \mu$. Anneau nerveux, diérides et pore excréteur respectivement à $155 \mu$, $270 \mu$ et $205 \mu$ de l'apex. 
Extrémité caudale pourvue sur le bord externe de sept paires de papilles: quatre pédonculées et trois sessiles; les quatre paires de papilles pédonculées soutiennent l'aile caudale ; les phasmides sont situées entre la sixième et la septième paire. Il y a en outre 5 papilles péri-cloacales : 3 sur la lèvre antérieure et 2 sur la lèvre postérieure. L'ornementation cuticulaire est composée de petits denticules chitineux (fig. 17, D). Spicules très inégaux et fins, le droit mesurant $1,7 \mathrm{~mm}$, le gauche $300 \mu$. Ouverture cloacale se trouvant à $190 \mu$ de l'extrémité postérieure.

Femelle: un peu plus grande que le mâle. Corps trapu, à extrémité antérieure large et postérieure effilée, mesurant $4,8 \mathrm{~mm}$ de longueur sur $350 \mu$ de largeur (fig. 17, E). Extrémité céphalique identique à celle du mâle. Esophage long, mesurant $2,5 \mathrm{~mm}$ (œsophage musculaire $170 \mu$ ). Anneau nerveux, diérides et pore excréteur respectivement situés à $210 \mu, 500 \mu$ et $450 \mu$ de l'apex.

Vulve à $1,45 \mathrm{~mm}$ de l'extrémité antérieure. Ovéjecteur long, dirigé vers l'arrière, comprenant un tube musculaire long de $950 \mu$, un réservoir à œufs de $570 \mu$ de longueur, se prolongeant par une trompe, aussi longue que le vagin, qui se divise en deux segments suivis chacun d'un utérus (fig. 17, F). Ces derniers se terminent en arrière par un réceptacle séminal bien différencié. Les deux ovaires sont enroulés autour de l'intestin postérieur. Eufs ovales, jaunâtres, embryonnés, de $50 \mu$ de longueur sur $30 \mu$ de largeur. Queue effilée, longue de $320 \mu$.

\section{Discussion.}

D'après le tableau dichotomique de Chabaud, 1956, nos spécimens appartiennent au genre Abbreviata (Travassos, 1920) et se rapprochent de A. leidyi (Walton, 1927), parasite de Varanus varius (Schaw) d'Australie, de A. natricis (Kreis, 1940), parasite de Natrix hypomelas Günther, de Nouvelle-Bretagne, et A. varani (Parona, 1889) sensu Ortlepp, 1922, parasite de Varanus monitor (L.) de Ceylan.

Pourtant nous n'avons pu identifier nos exemplaires à aucune des espèces précédentes, car ils possèdent un corps trapu et une tête de forme assez particulière.

Nous pensons que l'espèce est nouvelle et l'appelons Abbreviata brygooi n. sp., du nom du $\mathrm{D}^{\mathrm{r}}$ E. Brygoo, Directeur de l'Institut Pasteur de Madagascar.

10) ABBREVIATA LEGENDREI n. sp. (Physalopteridae).

\section{Matériel étudié.}

Plusieurs exemplaires mâles et femelles ont été récoltés dans l'estomac d'Iguanes appartenant à l'espèce Oplurus quadrimaculatus A. Dumer., de Ranohira-Isalo, qui portent les étiquettes $415 \mathrm{~F}, 1032 \mathrm{~F}, 1034 \mathrm{~F}, 1037 \mathrm{~F}, 1039 \mathrm{~F}$ et $437 \mathrm{G}$.

\section{Description.}

Mâle : corps grêle de 7,25 mm de longueur sur $250 \mu$ de largeur. Bouche délimitée par deux pseudo-lèvres latérales ; chacune porte une grosse dent externo-latérale et une petite interno-latérale (naissant à la base de la dent externo-latérale), plus deux petites doubles dents submédianes placées environ à mi-longueur de la bouche. Tout le long 

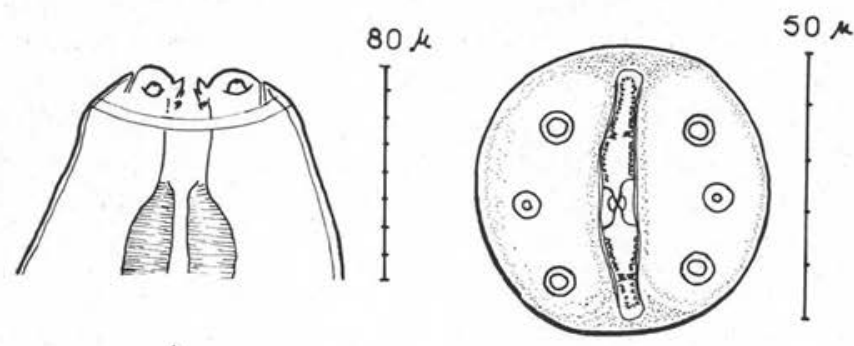

A

B
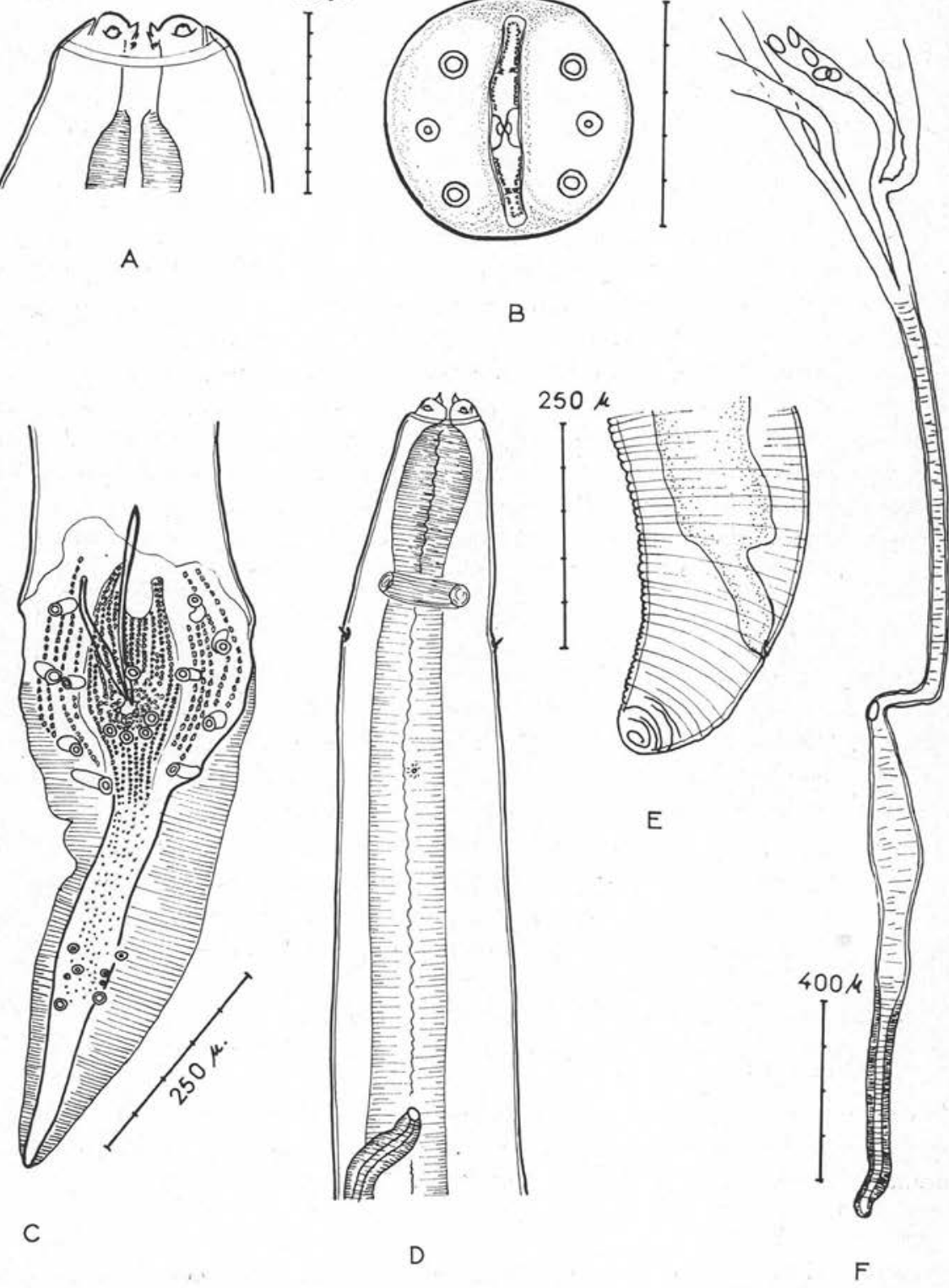

Fig. 18. - Abbreviata legendrei n. sp. A, extrémité antérieure du mâle, vue médiane. B, tête du mâle, vue apicale. C, extrémité postérieure du mâle, vue ventrale. $\mathrm{D}$, extrémité antérieure de la femelle, vue ventrale. E, extrémité postérieure de la femelle, vue latérale. F, ovéjecteur 
du bord buccal, y compris aux angles dorsaux et ventraux de la bouche, existe une série de petites dents. Deux papilles et une amphide sur chaque pseudo-lèvre (fig. 18, A, B). Longueur totale de l'œsophage : $2 \mathrm{~mm}$, avec portion musculaire de $190 \mu$ de
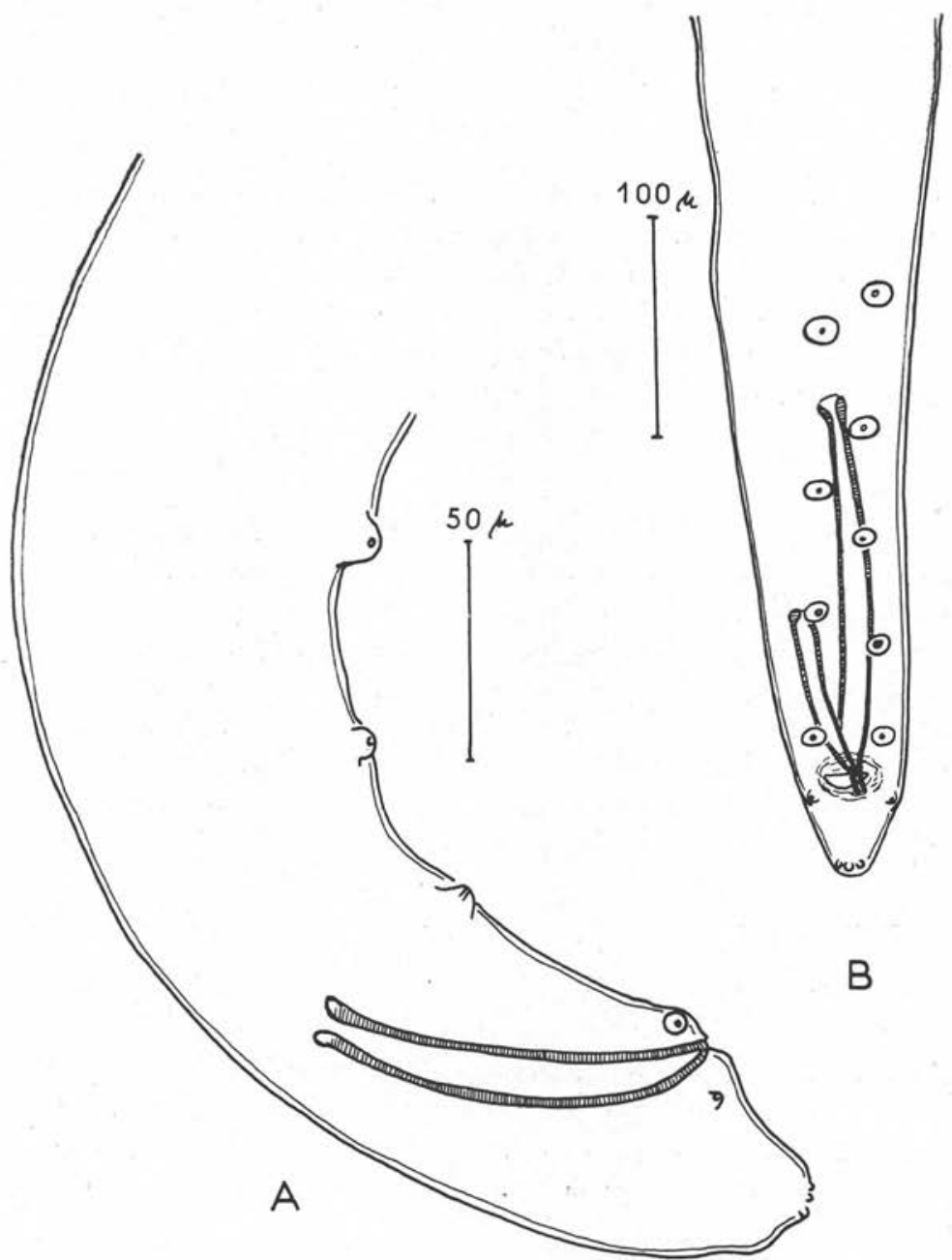

Fig. 19. - Madathamugadia opluri Chabaud, Anderson et Brygoo, 1959. Mâle. A, extrémité postérieure, vue latérale. B, extrémité postérieure, vue ventrale

long. Anneau nerveux, diérides et pore excréteur respectivement à $230 \mu, 280 \mu$ et $390 \mu$ de l'apex.

L'extrémité caudale, incurvée ventralement, est pourvue d'une bourse de $750 \mu$ de longueur. Cette bourse est formée par deux ailes caudales soutenues par cinq paires 
de papilles pédonculées: trois pré-cloacales et deux post-cloacales, plus onze papilles non pédonculées: une impaire pré-cloacale, quatre post-cloacales sur la lèvre postérieure du cloaque et six sur la queue. Entre la quatrième et la cinquième paire, deux phasmides se trouvant à $220 \mu$ de l'extrémité postérieure. Ornementation cuticulaire de la bourse composée de petites plaques chitinoïdes, régulièrement disposées (fig. 18, C). Spicules légèrement inégaux, fins, pointus, mesurant $155 \mu$ (spicule droit) et $225 \mu$ (spicule gauche). Cloaque à $530 \mu$ de l'extrémité postérieure.

Femelle: corps long et mince de $18 \mathrm{~mm}$ de longueur sur $210 \mu$ de largeur. Tête présentant la même structure que celle du mâle. Esophage musculaire mesurant $190 \mu$ de longueur, œsophage total 2,6 mm (fig. 18, D). Anneau nerveux, diérides et pore excréteur respectivement à $225 \mu 270 \mu$ et $410 \mu$ de l'apex.

Vulve à $800 \mu$ de l'extrémité antérieure. Ovéjecteur long et musculeux comprenant: le vagin de $500 \mu$ de longueur, un réservoir à œufs de $590 \mu$ de longueur, une trompe impaire, longue, se divisant en deux, puis en quatre utérus pleins d'œufs jaunâtres de $55 \mu$ de long sur $30 \mu$ de large (fig. 18, F).

\section{Discussion.}

L'espèce que nous venons de décrire présente les caractéristiques suivantes: bouche munie de dents externo-latérales submédianes et crête denticulée complète, spicules presque de la même taille.

D'après le tableau dichotomique de Chabaud, 1956, cette espèce se rapproche de A. ortleppi (Sandground, 1928), parasite de Chamaeleo dilepis (Leach) du Tanganyika, Afrique orientale et de A. antarctica (Linstow, 1899) sensu Irwin-Smith nec Ortlepp, parasite de l'Ophidien Acranthophis antarcticus Schaw, d'Australie.

Nous pensons qu'il s'agit d'une espèce nouvelle car on dénombre cinq paires de papilles pédonculées au lieu de quatre, et onze papilles sessiles au lieu de douze; la disposition des plaques chitinoïdes sur la bourse caudale est également différente.

Nous la dédions au $\mathrm{P}^{\mathrm{r}} \mathrm{R}$. Legendre, sous le nom de Abbreviata legendrei $\mathrm{n}$. sp.

11) MADATHAMUGADIA OPLURI * Chabaud, Anderson et Brygoo, 1959 (Onchocercidae).

Les parasites que nous avons trouvés dans la cavité générale de cinq Oplurus sebae (Dum. et Bibr.) provenant d'Ampijoroa possèdent les caractères du type ; cependant les auteurs décrivent cinq papilles pré-cloacales (trois à droite et deux à gauche), alors que certains de nos spécimens en portent neuf (quatre à droite et cinq à gauche) (fig. 19, A, B).

\section{B. - Caractères de la faune}

\section{1) Spécificité.}

La spécificité chez les Iguanes est beaucoup plus importante qu'elle ne l'est chez les Geckos. (Voir tableau II).

* L'orthographe adoptée pour l'hôte étant actuellement Oplurus, et non Hoplurus, nous amendons le nom de la Filaire en supprimant le $\alpha h$ » initial. 
D'après nos documents actuels, cinq espèces de Nématodes d'Iguanoidea sont étroitement spécifiques: les Oxyures Thelandros blanchardi et T. chabaudi; les Physaloptères Physalopteroides dollfusi et Abbreviata brygooi, et la Filaire Madathamugadia opluri.

Les Heterakides Strongyluris winteri et $S$. skrajabini n'ont été trouvés respectivement qu'une et deux fois chez des Oplurus.

Le Physaloptère Abbreviata legendrei est un parasite qui a été trouvé chez des Oplurus de différentes espèces, mais non chez d'autres Reptiles.

\begin{tabular}{|c|c|c|c|c|c|c|c|c|c|c|c|}
\hline \multirow[b]{2}{*}{$\begin{array}{c}\text { HôTES } \\
\text { Iguanoidea }\end{array}$} & \multicolumn{11}{|c|}{ NombRE DE SPÉCIMENS trouvés porteurs de: } \\
\hline & 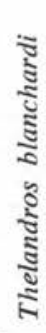 & 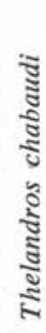 & 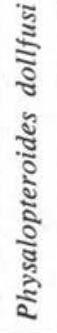 & 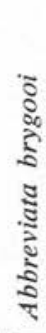 & 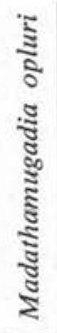 & 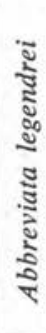 & 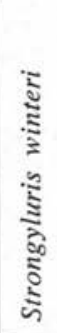 & 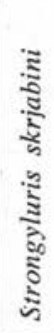 & 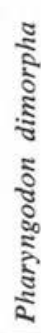 & 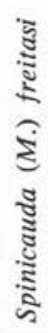 & 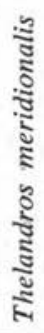 \\
\hline $\begin{array}{l}\text { Chalarodon } \\
\text { madagascariensis ...... }\end{array}$ & & & & & & & & & 3 & & \\
\hline Oplurus cyclurus ...... & & & & & & 1 & & & 3 & & 1 \\
\hline O. fierinensis ........ & & & & & & & & 1 & 2 & 1 & \\
\hline O. grandidieri $\ldots \ldots \ldots$ & & & & & & & 1 & & & & \\
\hline O. quadrimaculatus .... & 9 & 9 & & 2 & & 4 & & & & 1 & \\
\hline O. saxicola ........... & & & & & & 1 & & 1 & & & 1 \\
\hline O. sebae ............. & & & 4 & & 5 & & & & & & 1 \\
\hline Oplurus sp. .......... & & & & & & & & & 9 & & 1 \\
\hline $\begin{array}{l}\text { HôtES D'AUTRES } \\
\text { SUPERFAMILLES } \ldots \ldots \ldots\end{array}$ & $*$ & * & $*$ & * & $*$ & 一 & - & - & + & + & + \\
\hline
\end{tabular}

Tableau II. - Spécificité des Nématodes parasites d'Iguanoidea. Nous signalons: a) espèces fréquentes trouvées chez une seule espèce d'hôte $(*), b)$ espèces trouvées uniquement dans la superfamille des Iguanoidea $(-), c$ ) espèces trouvées chez les autres superfamilles de Sauriens 
L'Oxyuride Pharyngodon dimorpha a été retrouvé chez des Iguanes variés et chez les Caméléons.

L'Oxyuride Thelandros meridionalis et l'Hétérakide Spinicauda (Moaciria) freitasi ne sont pas spécifiques. Ils se trouvent chez tous les Sauriens malgaches.

\section{2) Endémisme et affinités.}

Les espèces étudiées sont endémiques à l'échelle spécifique, sauf la Filaire Madathamugadia opluri qui l'est à l'échelle générique.

Pharyngodon dimorpha semble avoir des affinités avec les espèces primitives décrites surtout en Australie.

Thelandros blanchardi, $T$. chabaudi et $T$. meridionalis appartiennent à un genre qui présente une grande unité dans le monde entier et dans lequel les affinités entre espèces sont difficiles à établir.

Spinicauda (Moaciria) freitasi peut être rapproché d'une espèce d'Amérique du Sud ou d'une espèce d'Afrique du Nord.

Strongyluris winteri a été rapproché des formes d'Afrique et d'Inde.

Strongyluris skrjabini, espèce très particulière par ses spicules, peut toutefois se rapprocher des formes de l'Inde, de Madagascar et du Brésil.

Physalopteroides dollfusi semble avoir des affinités avec des espèces d'Afrique du Sud, de Chine, d'Amérique du Sud et de Madagascar.

Abbreviata brygooi, extrêmement particulier par la forme de l'extrémité céphalique, présente des affinités avec les formes d'Australie, de Nouvelle-Bretagne (Archipel de Bismarck) et de Ceylan.

Abbreviata legendrei paraît avoir des affinités avec des espèces d'Afrique orientale et d'Australie.

Madathamugadia opluri, deuxième espèce du genre, est la forme la plus endémique rencontrée chez les Iguanes. De nombreux caractères, tels que la taille beaucoup plus grande, la constitution de l'ovéjecteur, l'œsophage musculaire plus court et nettement différencié du glandulaire, et la queue plus courte et conique, l'éloignent du genre australien Pseudothamugadia Lopez-Neyra, 1956.

Les affinités de cette faune sont encore plus variées qu'elles ne le sont chez les Geckos. Nous pouvons en donner une idée approximative en remarquant qu'au cours de la discussion des espèces nous avons cité l'Amérique du Sud deux fois, l'Afrique occidentale deux fois, l'Afrique orientale deux fois, l'Afrique du Sud, deux fois, l'Inde deux fois, l'Asie une fois, la région australienne cinq fois et l'Afrique du Nord une fois.

\section{3) Equilibre de la faune.}

La faune de Nématodes des Iguanes malgaches est riche, puisque une étude ne portant que sur une soixantaine de spécimens permet de rencontrer onze Nématodes différents.

Il est intéressant de la comparer à la faune des Nématodes d'Iguanes américains telle que nous la connaissons d'après les travaux de Caballero, 1941, Chitwood, 1934 
et Travassos, 1923. Il y a lieu de distinguer les espèces végétariennes et les espèces insectivores. Chez les premières, la faune d'Iguana tuberculata est particulièrement étudiée puisque l'on y cite (Skrjabin et collaborateurs, 1954) neuf espèces. Il y a une Filaire et huit espèces d'Oxyures constituant vraisemblablement un équilibre comparable à celui étudié par A. Petter chez les Tortues herbivores.

Chez les Iguanes insectivores, au contraire, très peu d'espèces sont signalées (Physaloptères, Strongylurides, Capillarides et quelques Oxyures généralement du genre Thelandros).

Les Iguanes malgaches sont tous insectivores et nous voyons qu'au niveau générique la composition de la faune parasitaire est très comparable à celle des Iguanes insectivores américains (la rareté des Capillaria a déjà été remarquée chez d'autres vertébrés de Madagascar).

Un problème particulier est posé par Oplurus quadrimaculatus : cette espèce est parasitée par l'association de deux Oxyures qui ne se retrouvent pas chez les autres Oplures et dont une au moins a des affinités nettes avec un Oxyure de Tortue herbivore. Cela pose un problème, non résolu. M. Ch.-P. Blanc, qui connaît particulièrement bien la biologie des Iguanes malgaches n'a pu mettre en évidence aucun élément (place systématique de l'Iguane, régime alimentaire, volume du côlon, localisation géographique) qui puisse expliquer pourquoi cette espèce à un parasitisme très différent de celui des autres Oplurus malgaches.

En dehors de cette particularité, la faune de Nématodes de Madagascar paraît donc bien diversifiée et ne présente pas d'anomalies fondamentales par rapport à celle des Reptiles analogues connus dans d'autres régions du monde.

\section{Nématodes de Chamaeleontoidea}

A la suite du travail de E.-R. Brygoo, 1963, nous avons poursuivi l'étude des Nématodes des Chamaeleonidae suivants: Brookesia superciliaris (Kuhl), B. ebenaui Boettger, Chamaeleo pardalis Cuvier, C. oustaleti Mocquard, C. brevicornis Günther.

\section{A. - Etude des espèces}

Parmi les vingt espèces connues comme parasites de Caméléons malgaches (Brygoo, 1963), nous en avons retrouvé cinq, plus une nouvelle, dont nous donnons la description détaillée. De plus, nous décrivons un spécimen mâle qui n'a pu être identifié à une espèce déterminée.

1) RHABDIAS GEMELLIPARA Chabaud, Brygoo et Petter, 1961 (Rhabdiasidae).

Cette espèce, parasite du poumon d'un Chamaeleo parsonii Cuvier, provenant de Périnet, fut retrouvée plusieurs fois chez $C$. brevicornis Günther, de différentes régions de l'Est de Madagascar.

Au cours de cette étude, nous l'avons identifiée une fois chez un Brookesia ebenaui d'Ampijoroa. Plus fréquemment nous l'avons trouvée chez les Zonosaures. 
2) MAXVACHONIA DIMORPHA, Chabaud et Brygoo, 1960 (Cosmocercidae).

L'espèce est connue comme parasite de l'estomac et de l'intestin moyen d'un Chamaeleo pardalis Cuvier, de Nossi-Bé.

Nous-même l'avons trouvée chez $C$. pardalis et $C$. oustaleti Mocquard, provenant également de Nossi-Bé (voir Chabaud, Caballero et Brygoo, 1965) ; plus tard, l'espèce a été identifiée de nouveau dans le rectum d'un $C$. pardalis d'Antalaha, chez un Zonosaure et chez un Mabuya.

3) SPINICAUDA (SPINICAUDA) INGLISI Chabaud et Brygoo, 1960 (Heterakidae).

Cette espèce, étudiée de l'intestin postérieur d'un Chamaeleo pardalis Cuvier, de Nossi-Bé, a été trouvée aussi chez $C$. oustaleti Mocquard, d'Andapa, d'Ampasimbe, de Meavatanana ; chez $C$. verrucosus Cuvier, d'Antsalova; chez $C$. guntheri Boulenger, $C$ brevicornis Günther et chez un Zonosaurus maximus Boulenger.

Nous-même l'avons trouvée chez $C$. oustaleti d'Ampijoroa et chez des Zonosaures variés.

4) STRONGYLURIS RADAODYI Chabaud et Brygoo, 1960 (Heterakidae).

L'espèce collectée dans l'intestin postérieur, les poumons et la cavité générale, est connue de divers Caméléons: Chamaeleo oustaleti Mocquard, de Doany, Antalaha et Andapa ; $C$. verrucosus, Cuvier, de Morafenobe et Mandritsara ; $C$. rhinoceratus Gray, de Majunga ; $C$. pardalis Cuvier ; $C$. guntheri Boulenger ; $C$. brevicornis Günther.

Nous l'avons retrouvée dans le rectum de C. pardalis de Nossi-Bé ; C. oustaleti d'Ampijoroa ; dans la cavité générale de C. brevicornis Günther, de Périnet, et chez un Zonosaure.

5) STRONGYLURIS RIOJAI n. sp. (Heterakidae).

\section{Matériel étudié.}

Le matériel, étiqueté $953 \mathrm{~F}$ et $961 \mathrm{~F}$, a été recueilli dans le rectum de deux Chamaeleo pardaiis Cuvier provenant de Nossi-Bé.

\section{Deseription.}

La cuticule présente ventralement de nombreuses rides irrégulières, longitudinales, sauf autour du pore excréteur qui occupe le centre d'une aire rectangulaire, à surface lisse. Pas d'ailes latérales. Chez la femelle, la cuticule présente à l'extrémité céphalique des papilles somatiques pédonculées que l'on ne trouve pas chez le mâle. La tête du mâle et celle de la femelle sont semblables, la bouche est entourée de trois lèvres triangulaires portant chacune une paire de petites papilles à l'apex (cycle interne). Deux papilles sur la lèvre dorsale et une sur les lèvres latéro-ventrales, plus deux amphides latérales (fig. 20, B).

Mâle : corps long de $8,4 \mathrm{~mm}$ et large de $460 \mu$. Tête de $58 \mu$, de diamètre. Le pharynx, long de $120 \mu$, se prolonge antérieurement par trois lobes, se terminant chacun par une dent petite et aiguë (fig. 20, C) ; la lumière du pharynx, à parois chitinoïdes, 


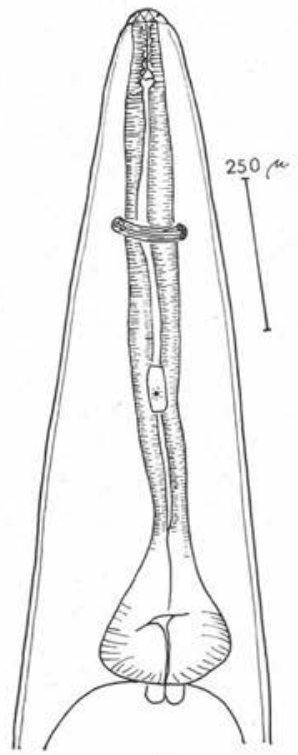

A
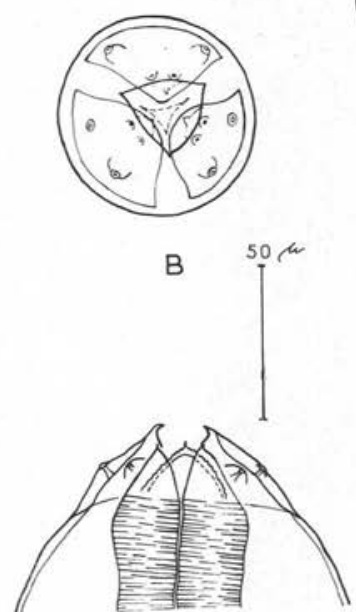

C

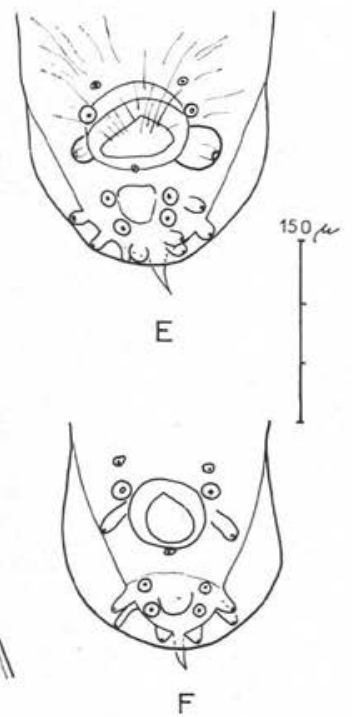

$\mathrm{F}$

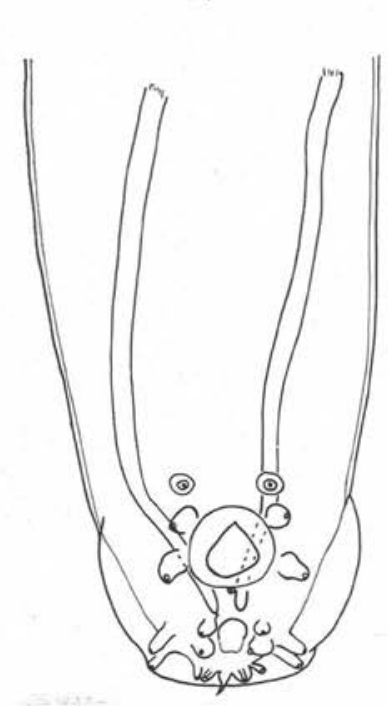

D
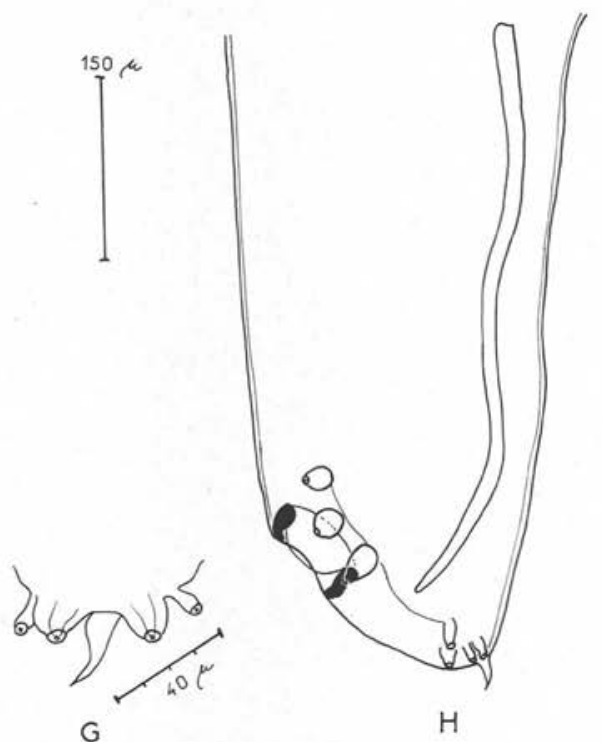

FIg. 20. - Strongyluris riojai n. sp. Mâle. A, extrémité antérieure, vue ventrale. B, tête, vue apicale. C, détail de l'extrémité antérieure, vue ventrale. D, E, F, extrémité postérieure, vues ventrales. G, détail des dernières paires de papilles caudales. $\mathrm{H}$, extrémité postérieure, vue latérale 

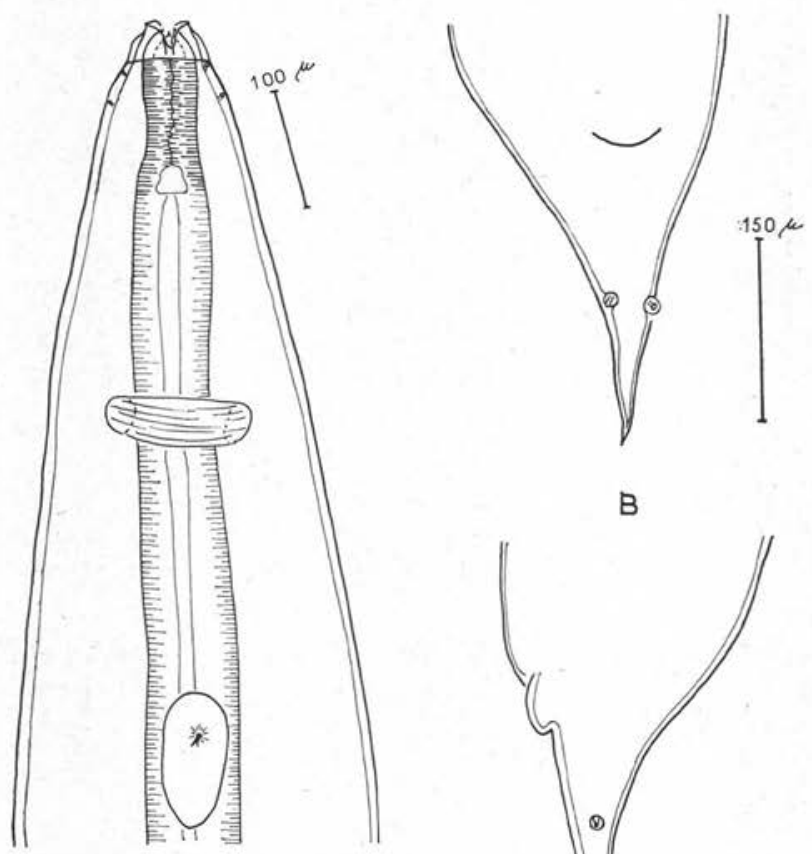

A
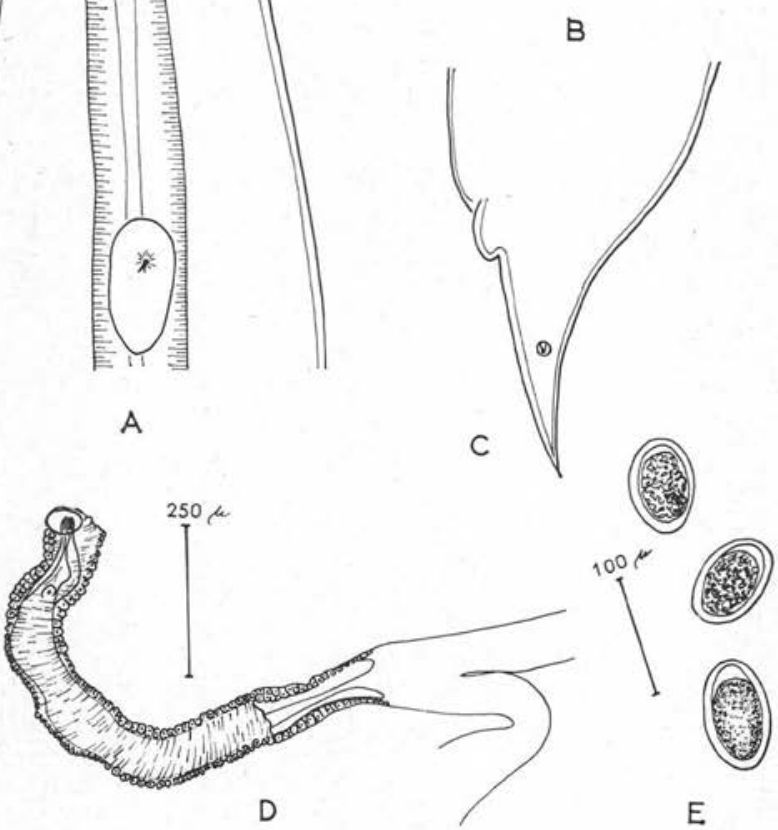

E.

Fig. 21. - Strongyluris riojai n. sp. Femelle. A, extrémité antérieure, vue ventrale. B, extrémité postérieure, vue ventrale. C, extrémité postérieure, vue latérale. D, ovéjecteur. E, œufs

s'élargit en arrière. L'œsophage, long de $970 \mu$ (bulbe compris) et large de $80 \mu$, se termine par un bulbe piriforme, long de $240 \mu$ et large de $210 \mu$ (fig. 20, A). Anneau nerveux à $370 \mu$ et pore excréteur à $630 \mu$ de l'apex. Les diérides n'ont pas été vues.

L'extrémité postérieure porte ventralement une ventouse presque circulaire de $70 \mu$ de diamètre, soutenue en profondeur par un anneau chitinoïde. Cette ventouse est située à $50 \mu$ du cloaque et à $120 \mu$ du bord postérieur du corps. Chez un autre spécimen, la distance ventouse-cloaque est de $40 \mu$ et la distance ventouse-extrémité posté- 
rieure de $130 \mu$. Les papilles génitales (fig. 20, D, G, H), au nombre de neuf paires plus une papille impaire, se répartissent de la façon suivante: trois paires pré-anales, la première étant peu pédonculée et antérieure à la ventouse, la seconde et la troisième, pédonculées et globuleuses, situées près des bords latéraux de la ventouse ; trois paires ad-anales, dont deux globuleuses sur les champs latéraux du cloaque et une digitiforme sur les bords latéraux du corps ; trois paires post-anales, dont la première est digitiforme et dorsale par rapport à la papille précédente; la deuxième est également digitiforme, mais grêle et petite, la troisième est globuleuse, petite, située de chaque côté de l'appendice caudal. Sur le bord postérieur du cloaque se trouve une encoche où est logée une toute petite papille. Au niveau de la première paire de papilles pré-cloacales, la cuticule se dilate, et les ailes caudales atteignent $25 \mu$ de largeur au niveau de la troisième paire de papilles pré-cloacales, pour constituer une bourse presque circulaire de $180 \mu$ de longueur sur $215 \mu$ de largeur au niveau de la ventouse. Spicules égaux, simples, longs de $450 \mu$ à $475 \mu$ et larges de $15 \mu$. Queue très courte et large, se terminant par un appendice caudal de $20 \mu$ de long.

Femelle : corps long de 14,2 mm et large de $310 \mu$. Extrémité céphalique de $65 \mu$ de diamètre. Lobes pharyngiens hauts de $20 \mu$. Pharynx de structure similaire à celle du mâle, long de $115 \mu$. L'œsophage, long de $1,01 \mathrm{~mm}$ et large de $70 \mu$, se termine par un bulbe sphérique de $210 \mu$ de diamètre. Anneau nerveux à $325 \mu$ et pore excréteur à $595 \mu$ de l'extrémité antérieure (fig. $21, \mathrm{~A}$ ).

La vulve, très postérieure, se trouve à $2 \mathrm{~mm}$ de la pointe caudale. L'ovéjecteur (fig. 21, D) se dirige vers l'arrière du corps, il comprend le vagin de $1,03 \mathrm{~mm}$ de longueur sur $100 \mu$ de largeur; les trompes se divisent en deux utérus, dont l'un se dirige vers la région antérieure, l'autre vers la région postérieure. Les œufs ovales (fig. 21, E), à coque épaisse, sont très nombreux, ils mesurent de 80 à $90 \mu$ de longueur sur 50 à $55 \mu$ de largeur. En avant de l'anus, le corps s'amenuise progressivement et se termine par une queue triangulaire, pointue, longue de $240 \mu$. A $115 \mu$ de la pointe caudale, deux phasmides peu saillantes sont bien visibles (fig. 21, B, C).

\section{Diseussion.}

Chez tous nos spécimens, la disposition des papilles génitales reste plus ou moins constante, mais leur aspect varie, car certaines d'entre elles s'hypertrophient et prennent une forme globuleuse.

Les espèces de Strongyluris les plus proches de la nôtre, parce qu'elles ont des spicules courts, sont $S$. bengalensis Chakravarty, 1936 et $S$. radaodyi Chabaud et Brygoo, 1960.

- S. bengalensis Chakravarty, 1936, parasite de l'Agamidae Calotes versicolor Brgr. de l'Inde, est différent par les caractères suivants: la ventouse est plus grande par rapport à la bourse caudale; les trois paires de papilles pré-cloacales se trouvent placées sur les bords latéraux de cette ventouse.

- S. radaodyi Chabaud et Brygoo, 1960, parasite de Chamaeleo oustaleti Mocquard, de Doany, Antalaha et d'Andapa (Madagascar), est différent par les caractères 


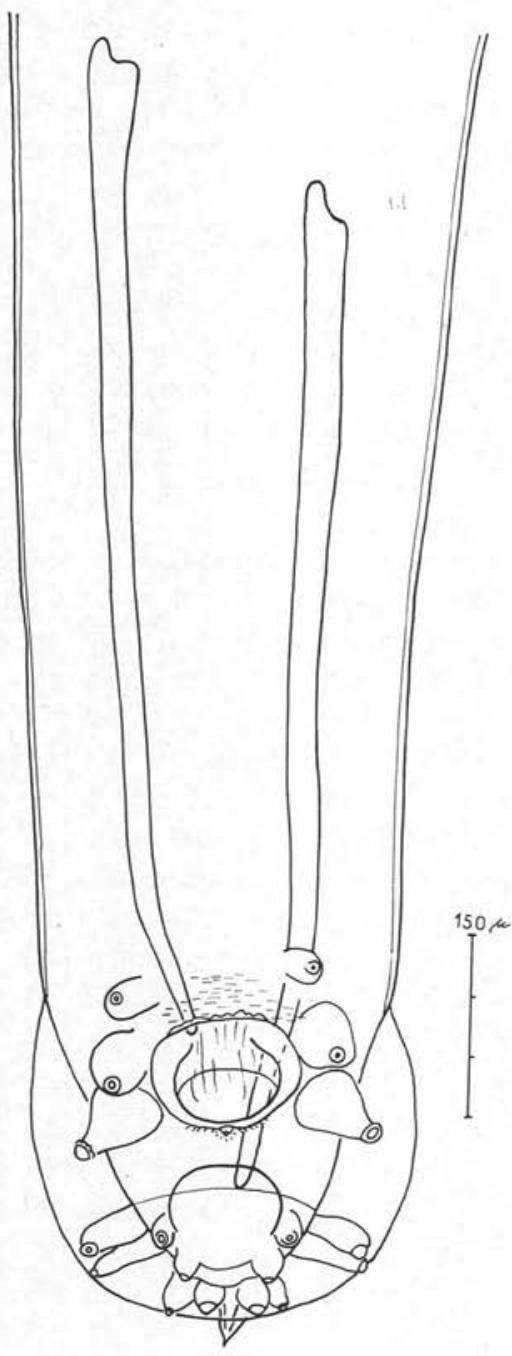

C

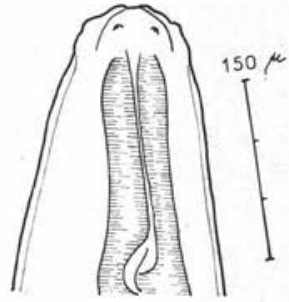

A

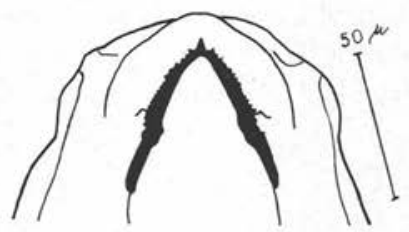

B

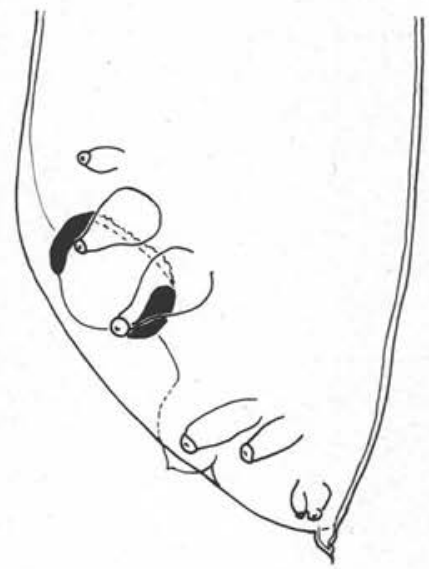

D

FIG. 22. - Strongyluris sp. Mâle. A, extrémité antérieure, vue dorsale. B, détail de l'extrémité anté rieure. C, extrémité postérieure, vue ventrale. D, extrémité postérieure, vue latérale

suivants : œsophage (pharynx et bulbe compris) plus long; bourse caudale allongée dans le sens longitudinal ; ventouse plus petite ; spicules plus longs $(640 \mu)$; œufs plus petits; queue de la femelle beaucoup plus longue. 
En raison de toutes ces différences, nous pensons donc qu'il s'agit d'une nouvelle espèce. Nous la nommons $S$. riojai n. sp., à la mémoire du $\mathrm{D}^{\mathrm{r}}$ Enrique Rioja Lobianco, Professeur à la Faculté des Sciences de Mexico et chercheur à l'Institut de Biologie de l’Université Nationale Autonome de Mexico.

6) STRONGYLURIS sp. (Heterakidae).

\section{Matériel étudié.}

Nous ne possédons qu'un seul spécimen mâle, qui porte l'étiquette $718 \mathrm{G}$, trouvé isolé dans le rectum d'un Chamaeleo brevicornis Günther provenant de Périnet. Nous en donnons les mensurations.

\section{Deseription.}

Longueur : 10,3 mm. Largeur : $600 \mu$. Cuticule dépourvue de papilles somatiques. Pas d'ailes latérales. Extrémité céphalique longue de $45 \mu$ et large de $77 \mu$ (fig. 22, A). Trois lobes pharyngiens dont le bord chitineux, libre, est dentelé et porte une petite dent triangulaire aiguë au sommet et deux dents latérales plus petites (fig. 22, B). Pharynx de $230 \mu$ de longueur sur $50 \mu$ de largeur. Esophage long de 1,65 mm et large de $100 \mu$ (corpus long de $1,37 \mathrm{~mm}+$ bulbe long de $280 \mu$, large de $275 \mu$ ). Anneau nerveux à $600 \mu$ de l'apex. Diérides et pore excréteur n'ont pas été observés.

Extrémité postérieure conique, avec un court appendice caudal de $50 \mu$ de long. Grande ventouse circulaire de $100 \mu$ de diamètre longitudinal sur $125 \mu$ de diamètre transversal, située à $230 \mu$ de l'extrémité postérieure et renforcée par un anneau chitinoïde en profondeur. Distance centre de la ventouse-lèvre antérieure du cloaque : $80 \mu$ Neuf paires de papilles génitales, plus une impaire. Ailes caudales prenant naissance au niveau de la première paire de papilles pré-cloacales (fig. 22, C, D). Extrémité postérieure (bourse caudale) de $300 \mu$ de long et $310 \mu$ de large. Spicules simples, égaux, bien chitinisés, de $820 \mu$ de long sur $30 \mu$ de large. Queue longue de $125 \mu$.

\section{Discussion.}

Le spécimen possède tous les caractères du genre Strongyluris. Il nous semble très proche de $S$. riojai décrit plus haut, cependant les dimensions beaucoup plus grandes, celles des spicules en particulier, nous font hésiter à le considérer comme appartenant à cette espèce. Nous préférons donc ne pas lui attribuer de détermination spécifique.

7) FOLEYELLA FURCATA (Linstow, 1899). (Onchocercidae).

Syn. : F. chamaeleonis Kreis, 1938. F. pigmentata Kreis, 1945.

Parasite de différents Caméléons malgaches, (voir Chabaud, 1959), nous l'avons trouvé cette fois-ci chez deux Chamaeleo pardalis Cuvier, de l'île de Nossi-Bé et chez un Chamaeleo brevicornis Günther, de Périnet. 


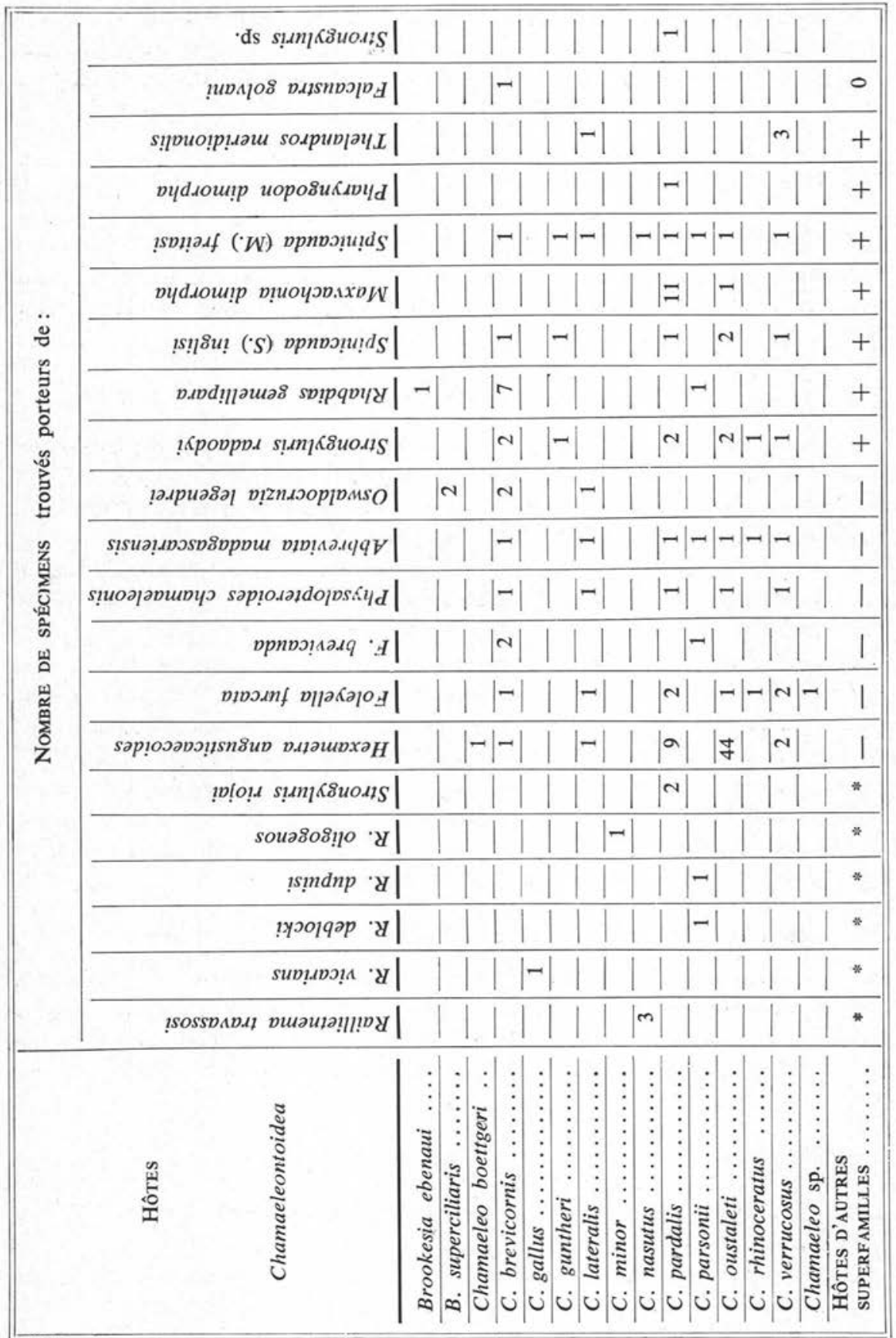

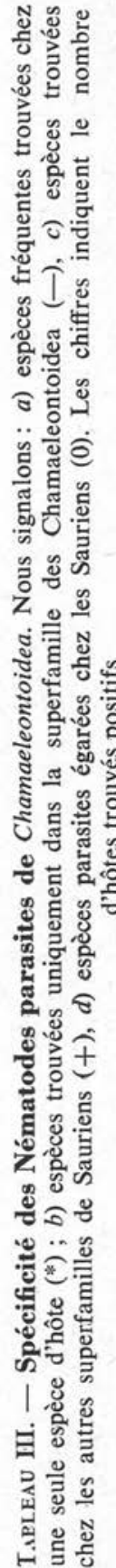




\section{B. - Caractères de la faune}

1) Spécificité.

La spécificité chez les Caméléons est moins stricte que chez les Iguanes. (Voir Tableau III).

Les cinq Cosmocercides du genre Raillietnema et l'Heterakide Strongyluris riojai pourraient avoir une spécificité très étroite, mais ils sont rares et la plupart n'ont été trouvés qu'une seule fois.

L'Ascaridide Hexametra angusticaecoides, les' deux Filaires: Foleyella furcata et F. brevicauda, et les deux Physaloptères: Physalopteroides chamaeleonis et Abbreviata madagascariensis ont été trouvés uniquement dans le genre Chamaeleo.

Le Strongle Oswaldocruzia legendrei n'a été trouvé que chez les Chamaeleonidae.

L'Heterakide Strongyluris radaodyi possède une large répartition dans le genre Chamaeleo. Nous l'avons trouvé égaré chez un Zonosaurus..

Le Rhabdiaside, Rhabdias gemellipara a été trouvé chez les Chamaeleonidae et fréquemment chez les Zonosaurus.

L'Heterakide Spinicauda (Spinicauda) inglisi est fréquent chez les Chamaeleo et chez les Zonosaurus.

Le Cosmocercide Maxvachonia dimorpha a été trouvé chez deux espèces de Chanaeleo, chez des Zonosaurus et chez des Mabuia. Son parasitisme chez Chamaeleo pardalis est remarquable. Cette espèce, pour des raisons écologiques qui nous sont inconnues, peut s'égarer chez les Gerrhosauridae et les Scincidae d'autres régions.

L'Heterakide Spinicauda (Moaciria) freitasi est très souvent rencontré dans le genre Chamaeleo, mais on le trouve aussi fréquemment chez les Uroplatus, Oplurus et Zonosaurus, notamment chez $Z$. madagascariensis et chez les Mabuia de l'Ile d'Europa.

Les espèces: Falcaustra golvani, Pharyngodon dimorpha et Thelandros meridionalis ont été considérées par Brygoo (1963) comme Nématodes parasites accidentels de Caméléons. Nous confirmons ce point de vue.

Falcaustra golvani est un parasite du genre Racophorus et d'autres Batraciens, accidentel chez les Caméléons.

Pharyngodon dimorpha est un parasite d'Iguanes, égaré chez les Caméléons. Nousmême l'avons retrouvé neuf fois sur vingt dissections de $O$. sebae; trois fois chez Chalarodon madagascariensis, et trois fois chez les Hemidactylus de l'Ile d'Europa.

Thelandros meridionalis est peu spécifique puisqu'on le trouve chez tous les Sauriens. Nous l'avons trouvé une fois chez les Geckonidae, deux fois chez les Oplurus, deux fois chez les Zonosaurus et quatre fois chez les Scincidae.

\section{2) Endémisme et affinités.}

A l'exception du Cosmocercidae Maxvachonia dimorpha qui est endémique à l'échelle de la sous-famille, toutes les espèces de Nématodes étudiées sont endémiques à l'échelle spécifique.

Le genre Rhabdias est connu de Batráciens et de Reptiles du monde entier, mais l'espèce $R$. gemellipara se rapproche de certaines formes africaines, en particulier de $R$. chamaeleonis et de $R$. entomelas de la région paléarctique. 
Oswaldocruzia legendrei se rapproche des espèces décrites des Agames et Caméléons de l'Est de l'Afrique.

Maxvachonia dimorpha, seule espèce du genre, se place dans la sous-famille Maxvachoninae.

Les espèces du genre Raillietnema ont des affinités avec l'espèce $R$. chamaeleonis d'Afrique orientale. Ce genre est connu du Brésil, de l'Afrique orientale et d'Australie, donc de l'Hémisphère austral.

Hexametra angusticaecoides appartient à un genre qui est connu chez les Ophidiens et Lacertiliens de l'Ancien et du Nouveau Monde.

Spinicauda (Spinicauda) inglisi appartient à un sous-genre formé par quatre espèces connues de Batraciens et de Reptiles variés d'Amérique du Sud, Australie, Afrique du Nord, Italie et Russie.

Spinicauda (Moaciria) freitasi appartient à un sous-genre connu par deux autres espèces parasites de Lézards du Brésil et d'Afrique du Nord.

Les deux espèces du genre Strongyluris sont très proches et possèdent des affinités avec les formes indiennes.

Abbreviata madagascariensis possède des affinités avec les formes africaines et australiennes.

Physalopteroides chamaeleonis se rapproche de $P$. impar décrit de Lacertiliens d'Afrique du Sud.

Les deux Foleyella appartiennent à un genre où les espèces parasites de Reptiles sont très homogènes. Les affinités avec l'espèce $F$. candezei d'Afrique sont grandes.

Au cours de la discussion des espèces, nous avons cité l'Amérique du Sud deux fois, la région éthiopienne six fois, l'Inde une fois, la région australienne trois fois, la région paléarctique trois fois et l'Afrique du Nord une fois. Donc les affinités sont variées, mais prédominent légèrement pour la région éthiopienne.

\section{3) Equilibre de la faune.}

Ainsi que l'a bien remarqué Brygoo, la composition générale de la faune de Nématodes de Caméléons malgaches est comparable à celle des Caméléons d'autres régions du Monde, exception faite pour le genre Maxvachonia. Les autres formes appartiennent aux mêmes genres ou sont proches de celles qui sont rencontrées chez les Caméléons d'Afrique ou d'Asie.

Les genres Rhabdias, Oswaldocruzia, Raillietnema, Hexametra, Spinicauda, Strongyluris, Abbreviata et Foleyella sont déjà connus d'autres Caméléons.

Le genre Physalopteroides est très proche du genre Thubunaea.

On notera la richesse en Cosmocercidae et Heterakidae puisque, sur dix-huit espèces reconnues comme parasites étroitement liés au genre Chamaeleo, onze appartiennent à ces deux groupes.

On notera également un grand nombre d'espèces égarées (Falcaustra golvani, Pharyngodon dimorpha et Thelandros meridionalis), mais il est difficile de savoir si cet élément est caractéristique de Madagascar, ou si, simplement, il est dû au fait que les autopsies ont été particulièrement nombreuses dans cette île.

(A SUIVRE). 